\title{
Phenotyping reproductive stage chilling and frost tolerance in wheat using targeted metabolome and lipidome profiling
}

\author{
Bo Eng Cheong ${ }^{1}$ - William Wing Ho Ho ${ }^{1,5} \cdot$ Ben Biddulph $^{3}$. Xiaomei Wallace ${ }^{2} \cdot$ Tina Rathjen $^{2}$. \\ Thusitha W. T. Rupasinghe ${ }^{4}$. Ute Roessner ${ }^{1,4} \cdot$ Rudy Dolferus $^{2}$ (1)
}

Received: 12 February 2019 / Accepted: 10 October 2019 / Published online: 20 October 2019

(c) The Author(s) 2019

\begin{abstract}
Introduction Frost events lead to A $\$ 360$ million of yield losses annually to the Australian wheat industry, making improvement of chilling and frost tolerance an important trait for breeding.

Objectives This study aimed to use metabolomics and lipidomics to explore genetic variation in acclimation potential to chilling and to identify metabolite markers for chilling tolerance in wheat.

Methods We established a controlled environment screening assay that is able to reproduce field rankings of wheat germplasm for chilling and frost tolerance. This assay, together with targeted metabolomics and lipidomics approaches, were used to compare metabolite and lipid levels in flag leaves of two wheat varieties with contrasting chilling tolerance.

Results The sensitive variety Wyalkatchem showed a strong reduction in amino acids after the first cold night, followed by accumulation of osmolytes such as fructose, glucose, putrescine and shikimate over a 4-day period. Accumulation of osmolytes is indicative of acclimation to water stress in Wyalkatchem. This response was not observed for tolerant variety Young. The two varieties also displayed significant differences in lipid accumulation. Variation in two lipid clusters, resulted in a higher unsaturated to saturated lipid ratio in Young after 4 days cold treatment and the lipids PC(34:0), PC(34:1), $\mathrm{PC}(35: 1), \mathrm{PC}(38: 3)$, and $\mathrm{PI}(36: 4)$ were the main contributors to the unsaturated to saturated ratio change. This indicates that Young may have superior ability to maintain membrane fluidity following cold exposure, thereby avoiding membrane damage and water stress observed for Wyalkatchem.

Conclusion Our study suggests that metabolomics and lipidomics markers could be used as an alternative phenotyping method to discriminate wheat varieties with differences in cold acclimation.
\end{abstract}

Keywords Cold tolerance $\cdot$ Flowering $\cdot$ Lipid $\cdot$ Metabolite $\cdot$ Phenotyping $\cdot$ Wheat

Electronic supplementary material The online version of this article (https://doi.org/10.1007/s11306-019-1606-2) contains supplementary material, which is available to authorized users.

Rudy Dolferus

rudy.dolferus@csiro.au

Bo Eng Cheong

cheongb@student.unimelb.edu.au

William Wing Ho Ho

whho@unimelb.edu.au

Ben Biddulph

ben.biddulph@dpird.wa.gov.au

Xiaomei Wallace

xiaomei.wallace@csiro.au

Tina Rathjen

tina.rathjen@csiro.au

Thusitha W. T. Rupasinghe

tru@unimelb.edu.au
Ute Roessner

u.roessner@unimelb.edu.au

1 School of BioSciences, The University of Melbourne, Melbourne, VIC 3010, Australia

2 CSIRO Agriculture \& Food, GPO Box 1700, Canberra, ACT 2601, Australia

3 Department of Primary Industries and Regional Development, 3 Baron Hay Court, South Perth, WA 6151, Australia

4 Metabolomics Australia, School of BioSciences, The University of Melbourne, Melbourne, VIC 3010, Australia

5 Melbourne Integrative Genomics, Schools of Mathematics and Statistics and of BioSciences, The University of Melbourne, Melbourne, VIC 3010, Australia 


\section{Introduction}

For important food crops, such as cereals, chilling and frost are major constraints to yield and productivity (Gray et al. 1997; Leff et al. 2004; Lobell and Gourdji 2012). Climate change affects atmospheric conditions, increasing the probability of weather extremes. This is likely to result in more frequent crop losses in the future (Lobell and Gourdji 2012). Warmer winters and more frequent spring frosts have increasingly affected wheat yields in China between 1961 and 2000 (Li et al. 2014, 2015). In Southern Australia, spring frosts have become significantly more frequent since 1960 and the overall length of the frost season has increased by 1 month, resulting in more frequent occurrences of frost damage to wheat crops (Crimp et al. 2015; Zheng et al. 2015). In Australia, spring wheat varieties are sown in autumn, flower in early spring and are harvested in late spring (Zheng et al. 2015). Radiant frosts in spring are caused by heat loss from the soil surface to the night sky on cold and dry days with clear skies. This leads to a rapid cooling of the crop canopy and associated frost damage if crops are at susceptible stages (Marcellos and Single 1975). The associated increase in risk of frost damage requires wheat cultivars with improved frost tolerance. Reoccurring frost events cost the Australian wheat industry an estimated A $\$ 360$ million of direct and indirect yield losses annually (March et al. 2015; Zheng et al. 2015).

Above zero degree temperatures (chilling; typically 10 to $0{ }^{\circ} \mathrm{C}$ ) cause an acclimation or adaptation response in plant tissues. Below zero degree temperatures (frosts) cause an additional challenge to plant tissues due to the formation of ice crystals. Quantitative and/or qualitative differences in the acclimation response during the chilling period may provide various degrees of frost tolerance. Wheat tolerates frosts during vegetative growth, but becomes more frost-sensitive during flowering in early spring. Chilling conditions during flowering can cause pollen sterility and grain loss in wheat (DemotesMainard et al. 1996; Rerkasem 1996; Subedi et al. 1998a, b, 2000; Chakrabarti et al. 2011). Exposure of plants to chilling conditions ("hardening") enhances freezing tolerance in a process called cold acclimation (Palta and Weiss 1993; Guy 1999; Tomashow 1999; Wanner and Junttila 1999). In wheat, cold acclimation during reproductive stages remains poorly characterised. Although most genes involved in cold acclimation are expressed after $24 \mathrm{~h}$ of exposure (Monroy et al. 2007; Kurepin et al. 2013), the full establishment of the response may require prolonged or repeated exposures to cold (Levitt 1980; Guy 1990; Ruelland et al. 2009). Cold acclimation can be lost during warmer fluctuating winter conditions, but it remains unclear whether re-acclimation can occur following deacclimation (Rapacz 2002; Kalberer et al. 2006; Vitamvas and Prasil 2008), particularly after crops transition into reproductive stages. Loss of cold acclimation during spring makes wheat crops particularly vulnerable to unexpected frosts (Frederiks et al. 2011, 2012). Cold temperatures, in combination with increasing day-length, accelerate the transition from vegetative to reproductive growth as the vernalization and photoperiod requirements are met (Fowler et al. 2001; Limin and Fowler 2006). Proper management of sowing time and phenology of wheat (flowering time, response to day-length) minimise frost damage, but the unpredictability of frost events requires genetic improvement in frost tolerance to manage yield stability (Limin and Fowler 2006; Zheng et al. 2015), particulary in Southern Australia where the length of the frost season is increasing (Crimp et al. 2015) and crop development can not be delayed further.

Induction of cold acclimation and freezing tolerance involves physiological and biochemical changes in plants (Wanner and Junttila 1999). Cold-tolerant plants have a higher proportion of unsaturated fatty acids in the plasma membrane and are better at maintaining membrane fluidity at lower temperatures (Vigh et al. 1979; Steponkus et al. 1993; Bohn et al. 2007). Plants tend to increase the degree of fatty acid unsaturation and the content of phospholipids when they are exposed to low, non-freezing temperatures (Welti et al. 2007). In Arabidopsis, phophatidylcholines (PC), phosphatidylethanolamines (PE), phosphatidylglycerols (PG) and monogalactosyldiacylglycerols (MGDG) containing two polyunsaturated acyl species, including 36:5 (18:2/18:3) and 36:6 (di-18:3), increased during cold acclimation (Welti et al. 2002). Chilling conditions also lead to the accumulation of osmolytes, compatible solutes or cryoprotectants such as sugars (fructose, glucose, trehalose, raffinose) and amino acids or amines such as proline and gamma-aminobutyric acid (GABA). This response is not specific to chilling and is also induced by other stressors that cause osmotic stress (e.g., drought, salinity, heat; Beck et al. 2007). Apart from playing a role in compensating for water loss during osmotic adjustment, osmolytes are also important for protecting membrane integrity and scavenging of reactive oxygen species (Guy 1999; Wanner and Junttila 1999; Smallwood and Bowles 2002; Valluru et al. 2008; Javadian et al. 2010; Verslues et al. 2006; Janmohammadi 2012). Cold acclimation in spring and winter Triticum monococcum lines affects the steady-state levels of phytohormones, including abscisic acid (ABA), salicylic acid, ethylene, jasmonic acid, gibberellins, cytokinins and auxin (Horvath et al. 2007; Machakova et al. 1989; Vanková et al. 2014). Increased ABA levels are correlated with cold-induced water deficit in plants. ABA is implicated in plasma membrane lipid alterations during cold stress (Bohn 
et al. 2007). ABA induces the expression of phospholipase D (AtPLD) in Arabidopsis, causing hydrolysis of structural phospholipids (Zhang et al. 2005; Meijer and Munnik 2003).

Studying quantitative and qualitative differences in metabolite and lipid accumulation during cold acclimation is an important tool to understand chilling and frost tolerance in wheat. Targeted metabolomics and lipidomics using gas/liquid chromatography, coupled to a triple-quadruple MS (GC/LC-QqQ-MS) approach offers higher sensitivity, selectivity, reproducibility and robust quantification over a broad dynamic range (Douglas 2009; Sumner et al. 2015; Jorge et al. 2016). Dias et al. (2015) were able to quantify 76 primary metabolites (sugars, organic acids and amino acids/ amines) in two chickpea cultivars with contrasting responses to salinity using GC- and LC-QqQ-MS. Natera et al. (2016) reported the quantification of 63 phospholipids in the roots of two barley genotypes with contrasting responses to salinity using LC-QqQ-MS. We therefore applied these approaches to study the cold acclimation response in wheat.

Reliable phenotyping for chilling and frost tolerance in the field is challenging due to unpredictability, variability in severity, duration, and timing of these events. In this study we established a reliable controlled environment (CE) phenotyping method to discriminate genetic variation in cold and frost tolerance in wheat. This CE phenotyping method, together with targeted metabolomics and lipidomics approaches, were then used to identify quantitative and qualitative differences in metabolite and lipid accumulation in two wheat varieties with differential chilling-tolerance. As starting tissue we used flag leaves from wheat plants harvested at the young microspore (YM) stage of pollen development, the reproductive stage with highest sensitivity to various abiotic stresses -including cold (Dolferus et al. 2013). The results indicate that two Australian spring wheat varieties with contrasting chilling tolerance (coldtolerant Young and cold-sensitive Wyalkatchem) differed significantly in the accumulation of metabolites (amino acids, osmolytes) and levels of unsaturated versus saturated membrane lipids, indicating that metabolite profiling could be used to select wheat varieties with differences in cold acclimation.

\section{Materials and methods}

\subsection{Controlled environment wheat growing, cold treatment and sample collection}

Wheat varieties Wyalkatchem (cold-sensitive) and Young (cold-tolerant) used in this study were obtained via the National Frost Initiative, Australia (http://www.nvtonline. com.au/frost/). Seeds were sown in trays $(36 \times 25 \times 13 \mathrm{~cm}$, $\mathrm{L} \times \mathrm{W} \times \mathrm{H} ; 15$ plants/tray) filled with soil ( $100 \%$ composted soil, containing $1 \mathrm{~g} \mathrm{l}^{-1}$ fertilizer: $14.4 \% \mathrm{~N} / 6.6 \% \mathrm{P} / 5 \% \mathrm{~K}$ ). Plants were grown in the glasshouse under natural lighting conditions and controlled temperature regime $\left(24 / 16{ }^{\circ} \mathrm{C}\right.$, $\mathrm{L} / \mathrm{D}$ ); watering occurred once daily. The young microspore (YM) stage of pollen development was determined using auricle distance measurements (Ji et al. 2010; Dolferus et al. 2013). The Zadoks scale was used to determine growth stages other than YM (Zadoks et al. 1974). Cold treatments were carried out at the YM stage in a Conviron PGC 20 growth chamber. The treatment cycle consisted of $12 \mathrm{~h}$ incubation at $21{ }^{\circ} \mathrm{C}$ in the light, followed by a linear cooling gradient descending from 21 to $-3^{\circ} \mathrm{C}$ over a period of $4 \mathrm{~h}$, then followed by a continuous cold period at $-3{ }^{\circ} \mathrm{C}$ over $8 \mathrm{~h}$ in the dark (12/12 light/dark cycle, using $400 \mu \mathrm{mol} \mathrm{m}^{-2} \mathrm{~s}^{-1}$ light intensity; see Fig. S1a). The whole experiment was run over four consecutive days and samples were harvested in the morning immediately after a cold treatment at day one ( $\mathrm{TP}_{1}$ — short cold stress), day four $\left(\mathrm{TP}_{3}\right.$ - prolonged cold stress), and corresponding day-time samples were harvested $6 \mathrm{~h}$ into the normal temperature $\left(21^{\circ} \mathrm{C}\right)$ light cycle after day one and day four respectively $\left(\mathrm{TP}_{2}\right.$ and $\mathrm{TP}_{4}$; see Fig. S1f). One tray of plants was used as untreated control to harvest $\mathrm{T}_{0}$ samples. In the trays for stress treatments, tillers that reached the YM stage were tagged before cold-treatment. Three flag leaves were harvested from three different tagged plants at each of the time points $\left(\mathrm{TP}_{0}\right.$ to $\left.\mathrm{TP}_{4}\right)$ and pooled as one replicate for metabolite measurements. For each time point, we harvested four biological replicates per variety $(n=4)$ for all the metabolite and lipid analyses, except for phytohormone analyses where three replicates were used $(n=3)$. For sterility measurements, plants were returned to the glasshouse after cold treatment, and spike grain number of tagged tillers was determined at maturity.

\subsection{Analysis of sugars, organic acids, amino acids, amines and phytohormones}

All chemicals and solvents for metabolite measurements were purchased from Sigma-Aldrich (Australia) and were of analytical or mass spectrometry grades. For sugars, organic acids and amines, tissue extraction was performed according to Dias et al. (2015), with some modifications. Aliquots of frozen leaf material $(50 \mathrm{mg}$ per replicate $\times$ four replicates) were weighed into Cryomill tubes (Precellys 24, Bertin Technologies). Subsequently, $500 \mu \mathrm{l}$ of methanol containing $4 \%$ of internal standard (from a stock solution containing $0.5 \mathrm{mg} \mathrm{ml}^{-1}$ of ${ }^{12} \mathrm{C}_{6}$-sorbitol and $0.5 \mathrm{mg} \mathrm{ml}^{-1}{ }^{13} \mathrm{C}_{5^{-}}{ }^{15} \mathrm{~N}-\mathrm{L}-$ valine) was added to the samples, followed by vortexing for $30 \mathrm{~s}$ and homogenization at $-10{ }^{\circ} \mathrm{C}$ using a Cryomill $(3 \times 45 \mathrm{~s}$ at $6100 \mathrm{rpm})$. The samples were then extracted for $15 \mathrm{~min}$ at $30{ }^{\circ} \mathrm{C}$ in a thermomixer at $850 \mathrm{rpm}$, and subsequently centrifuged for $5 \mathrm{~min}$ at $4{ }^{\circ} \mathrm{C}$ at $13,000 \mathrm{rpm}$. The supernatants were transferred into new tubes, and $500 \mu \mathrm{l}$ of 
water containing $0.2 \%$ formic acid was added into the Cryomill tubes containing the previously ground tissue pellet. The samples were vortex-mixed for $30 \mathrm{~s}$, and centrifuged at $13,000 \mathrm{rpm}$ for $5 \mathrm{~min}$ at $4{ }^{\circ} \mathrm{C}$. The supernatants were then transferred and combined with the methanolic extracts from the previous centrifugation. A $200 \mu \mathrm{l}$ of dichloromethane was added to the combined supernatants to separate chlorophylls. The combined supernatants were vortexed, centrifuged at $13,000 \mathrm{rpm}$ for $2 \mathrm{~min}$ at $4{ }^{\circ} \mathrm{C}$. The supernatants were taken and stored at $-80{ }^{\circ} \mathrm{C}$ for subsequent sugars, organic acids and amine analyses.

For the analysis of sugars and organic acids, $5 \mu \mathrm{l}$ and $125 \mu \mathrm{l}$ aliquots of the supernatants was transferred into new glass vial inserts, and dried in vacuo for sugars and organic acids analyses using GC-QqQ-MS. Prior to the GC-QqQMS analysis, the dried extracts were derivatized with methoxyamine hydrochloride in pyridine and bis-(trimethylsilyl)trifluoroacetamide (BFTFA) as described by Dias et al. (2015). Briefly, All samples were re-dissolved in $20 \mu \mathrm{l}$ of $30 \mathrm{mg} \mathrm{ml}^{-1}$ methoxyamine hydrochloride in pyridine and derivatized at $37{ }^{\circ} \mathrm{C}$ for $120 \mathrm{~min}$ with mixing at $500 \mathrm{rpm}$. The samples were incubated for $30 \mathrm{~min}$ with mixing at $500 \mathrm{rpm}$ after addition of $20 \mu \mathrm{l}, \mathrm{O}$-bis-(trimethylsilyl)trifluoroacetamide (BSTFA). Each derivatized sample was allowed to rest for $60 \mathrm{~min}$ prior to injection. Later, the derivatized samples (injection volume of $1 \mu \mathrm{l}$ for each sample) were injected into a GC-QqQ-MS system comprising of a Gerstel 2.5.2 Autosampler, a 7890A Agilent gas chromatograph and a 7000 Agilent triple-quadruple MS (Agilent Santa Clara, USA) with an electron impact (EI) ion source. The instrument settings were the same as described by Dias et al. (2015). For calibration and quantification of sugars and organic acids in the flag leaf samples, we used serial concentrations of calibration authentic standards (Table S1), including 24 sugars (sugars, sugar phosphates, sugar acids and sugar alcohols) and 19 organic acids, derivatized and subjected to GC-QqQ-MS analysis-as described by Dias et al. (2015).

For the amino acid and amine analyses with LC-QqQMS, $10 \mu \mathrm{l}$ of supernatants were transferred into new glass vial inserts. The amino acids and amines in the supernatants were then derivatized with 6-aminoquinolyl- $N$-hydrosysuccinimidyl carbamate (AQC) reagent as described in Boughton et al. (2011) and Dias et al. (2015). Briefly, 10 $\mu \mathrm{l}$ samples were added to $70 \mu \mathrm{l}$ of borate buffer $(200 \mathrm{mM}$, $\mathrm{pH} 8.8$ at $25^{\circ} \mathrm{C}$ ) containing $10 \mathrm{mM}$ TCEP, $1 \mathrm{mM}$ ascorbic acid and $50 \mu \mathrm{M} 2$-aminobutyrate. The resulting solution was vortexed before adding $20 \mu \mathrm{l}$ of 6-aminoquinolyl$\mathrm{N}$-hydrosysuccinimidyl carbamate (AQC) reagent [ $200 \mathrm{mM}$ dissolved in $100 \%$ acetonitrile (ACN)] and then immediately vortexed. The samples were heated with shaking at $55{ }^{\circ} \mathrm{C}$ for $10 \mathrm{~min}$, then centrifuged at $13,000 \mathrm{rpm}$ at room temperature and transferred to HPLC vials containing inserts
(Agilent, springless glass inserts, $250 \mu \mathrm{l}$ ) prior to injection. The derivatized samples (injection volume of $1 \mu \mathrm{l}$ for each sample) were immediately injected into a LC-QqQ-MS system comprising of an Agilent 1200 LC-system coupled to an Agilent 6410 Electrospray Ionization-Triple QuadrupleMS. The setting of the LC-MS instrument were as described by Dias et al. (2015). For calibration and quantification of amino acids and amines in the flag leaf samples, a series of concentrations of calibration authentic standards (Table S1), comprising of 29 amino acids and amines mixed with sulfurcontaining compound solution were prepared, derivatized and subjected to LC-QqQ-MS analysis same as described by Dias et al. (2015).

Seven phytohormones and phytohormone-related compounds [salicylic acid (SA), jasmonic acid (JA), jasmonoylisoleucine (JA-ile), indole-3-acetic acid (IAA), indole3-carboxylic acid (ICA), indole-3-butyric acid (IBA) and 2-cis-4-trans-abscisic acid (ABA)], as well as three organic acids which are precursors for phytohormones [(benzoic acid (BA), trans-cinnamic acid (CA) and 12-oxo-phytodienoic acid (OPDA) were analysed in this study. Extraction for phytohormone analysis was carried out using a slightly modified procedure of Cao et al. (2017). Briefly, the flag leaf samples were ground with pestle and mortar in liquid nitrogen and frozen immediately. A $100 \mathrm{mg}$ of sample was weighed for each of the samples and transferred to a dried $2 \mathrm{ml}$ centrifuge tube that was pre-washed with MS grade methanol. $1 \mathrm{ml}$ of extraction solvent, containing $956 \mu \mathrm{l}$ of $70 \%$ methanol and $44 \mu \mathrm{l}$ of $8.3 \mu \mathrm{g} \mathrm{ml}^{-1}$ internal standard mixtures $\left(\mathrm{d}_{2} \mathrm{IAA}, \mathrm{d}_{5} \mathrm{BA}, \mathrm{d}_{6} \mathrm{ABA}, \mathrm{d}_{6} \mathrm{SA}, \mathrm{d}_{7} \mathrm{CA}, \mathrm{H}_{2} \mathrm{JA}\right)$, was added to the sample, then vortexed and agitated at $4{ }^{\circ} \mathrm{C}$, and spun at $1400 \mathrm{rpm}$ for $30 \mathrm{~min}$. The samples were then centrifuged at $16,100 \times g$ for $10 \mathrm{~min}$. The supernatants were transferred to fresh centrifuge tubes. The residues were reextracted and centrifuged with $500 \mu \mathrm{l}$ of extraction solvents (without internal standards). Finally, the first and second supernatant was combined and kept at $-80{ }^{\circ} \mathrm{C}$ until further derivatization steps using methyl-chloroformate (MCF). The subsequent derivatization steps were carried out according to Rawlinson et al. (2015), with some modifications. While Rawlinson et al. used GC coupled with single MS, our analysis used triple quadruple GC-MS (GC-QqQ-MS) with newly developed multiple reaction monitorings (MRMs) which were developed for each of the measured phytohormone and organic acids. Briefly, $600 \mu \mathrm{l}$ of the supernatant for each sample was taken, dried down and re-dissolved with $160 \mu \mathrm{l}$ methanol. Then, $8.3 \mu \mathrm{g} \mathrm{ml}{ }^{-1}$ of ${ }^{13} \mathrm{C}_{5^{-}}{ }^{15} \mathrm{~N}-\mathrm{L}$-Valine (another internal standard) was added. $34 \mu \mathrm{l}$ of pyridine was then added to the mixture, followed by vortexing vigorously for $25-30$ s. $200 \mu \mathrm{l}$ of $1 \% \mathrm{NaOH}$ solution was then added to the mixture. This $\sim 400 \mu \mathrm{l}$ mixture was then derivatized with 20 $\mu \mathrm{l}$ of methyl chloroformate (MCF) and vortexed vigorously for 25-30 s. Another $20 \mu \mathrm{l}$ of methyl chloroformate (MCF) 
was added, followed by vortexing. $400 \mu \mathrm{l}$ of chloroform was then added to the mixture, vortexed again for $10 \mathrm{~s}$, followed by an addition of $400 \mu \mathrm{l}$ of $50 \mathrm{mM}$ sodium bicarbonate. The mixture was vortexed for 10-15 s and centrifuged for $30 \mathrm{~s}$ at $16,100 \times g$. The upper aqueous layer was discarded and the bottom organic layer was taken $(1 \mu \mathrm{l}$ for each sample) and injected into a GC-QqQ-MS system comprising a Gerstel 2.5.2 Autosampler, a 7890A Agilent gas chromatograph and a 7010 Agilent triple-quadrupole MS (Agilent, Santa Clara, USA) with an electron impact (EI) ion source. More details for the preparation of calibration and internal standards, extraction, derivatization steps and GC-QqQ-MS instrument settings can be found in Supplementary Data S1 (Methodology for phytohormone analysis).

\subsection{Analysis of lipid content}

The extraction of lipids was carried according to Folch et al. (1957), with modifications. Briefly, $30 \mathrm{mg}$ freshly frozen flag leaf samples were homogenized in $500 \mu \mathrm{l}$ of a 2:1 methanol:chloroform mixture using a Cryomill (Precellys 24 , Bertin technologies) for $3 \times 45 \mathrm{~s}$ at $6100 \mathrm{rpm}$ $\left(-10{ }^{\circ} \mathrm{C}\right)$. The extracts were shaken at $750 \mathrm{rpm}$ for $15 \mathrm{~min}$ at $30^{\circ} \mathrm{C}$, and centrifuged at $13,000 \mathrm{rpm}$ for $15 \mathrm{~min}$. The supernatants were transferred to new tubes, while $500 \mu \mathrm{l}$ of 2:1 methanol:chloroform mixture was added again to each of the remaining residue, vortexed and centrifuged at $13,000 \mathrm{rpm}$ for $15 \mathrm{~min}$. The supernatants were combined with the previous supernatants. The combined supernatants were dried down in vacuo and re-constituted in $100 \mu \mathrm{l}$ of 1:1 butanol:methanol for the subsequent LC-QqQ-MS analysis using an Agilent 1200 LC-system coupled to an Agilent 6410 Electrospray Ionization-Triple Quadruple-MS system. An injection of $5 \mu$ of each total lipid extract was chromatographically separated on an Ascentis Express RP-Amide $50 \times 2.1 \mathrm{~mm}, 2.7 \mu \mathrm{m}$ HPLC column (Sigma-Aldrich, Castle Hill, NSW) using an 8 min gradient from $0 \%$ A to $100 \%$ B, which was then held for 2 min and followed by a 4 min column re-equilibration with a flow rate of $0.18 \mathrm{ml} \mathrm{min}^{-1}$. The mobile phases were: A, $10 \mathrm{mM}$ ammonium formate in water: methanol: tetrahydrofuran (50: 20: 30, v/v/v); B, $10 \mathrm{mM}$ ammonium formate in water: methanol: tetrahydrofuran (5: 20: $75, \mathrm{v} / \mathrm{v} / \mathrm{v})$. Lipid species were identified and quantified based on multiple reaction monitorings (MRMs) established using external lipid standards and references from the LIPIDMATCH database (https://github.com/GarrettLab-UF/ LipidMatch/releases/tag/v2.0.2) as listed in Table S2, with a 5-20 ms dwell time for the simultaneous measurements of up to 100 compounds. We used optimised parameters for capillary $(4000 \mathrm{~V})$, fragmentor $(60-160 \mathrm{~V})$ and collision voltages $(20-40 \mathrm{~V})$. In all cases, the collision gas was nitrogen with a flow rate of $71 \mathrm{~min}^{-1}$. The external lipid standards used (Table S2) were lysophosphatidylcholine
LPC(17:0), phospatidylcholine PC(34:1), phosphatidylethanolamine PE(34:0), phosphatidylglycerol PG(34:1), phosphatidylinositol PI(36:2), phosphatidic acid (PA) and phosphatidylserine PS(34:0). Detected lipid species are annotated as follows: lipid class designation (total number of carbon atoms in the fatty acid chains: total number of double bonds in the fatty acid chains).

\subsection{Data processing and statistical analysis of metabolite data}

Raw metabolite and lipid data were processed and analyzed using Quantitative Analysis MassHunter Workstation software for QQQ (Agilent Technologies, Santa Clara, CA, USA). The level of identification was carried out based on the Metabolomics Standards Initiative (MSI) requirements (Sumner et al. 2007). For all measured metabolites (sugars, organic acids, amines and phytohormones), absolute quantities were determined while the concentration unit was expressed as picomole/mg of fresh weight (Table S3), using MSI Level 1 as the identification was based on Multiple Reaction Monitorings (MRMs) established using authentic standards (Table S1). Meanwhile, for lipids, responses were normalized to $\mathrm{mg}$ fresh weight (Table S4). Although single authentic lipid species were used for each of the phospholipid class, the identification of individual lipid species was based on the MRM experiment and retention time (MSI Level 2, Table S2). The processed metabolite and lipid data were first subjected to multiple comparison statistical analyses using Analysis of Variance (ANOVA), with a false discovery rate (FDR)-adjusted $p$ value of 0.05 and using the Benjamini-Hochberg method (1995) to determine the metabolites and lipids that changed significantly across all time points or over the 4-day cold stress period, followed by the Tukey's honestly significant difference (HSD) post hoc test in order to determine which pair-wise combinations showed significant differences for both primary metabolites and lipids using Graphpad Prism 7.0 (GraphPad Software, La Jolla California USA). Next, we determined which metabolites/lipids responded specifically to short cold stress $\left(\mathrm{TP}_{1}\right)$, prolonged cold stress $\left(\mathrm{TP}_{3}\right)$, diurnal fluctuations when the plants were exposed to normal day-time temperature of $21{ }^{\circ} \mathrm{C}$ for $6 \mathrm{~h}\left(\mathrm{TP}_{2}\right.$ and $\left.\mathrm{TP}_{4}\right)$. We therefore performed pairwise comparisons of the selected two groups $\mathrm{TP}_{1}$ versus $\mathrm{TP}_{0}$ (for short cold stress), $\mathrm{TP}_{3}$ versus $\mathrm{TP}_{1}$ (for prolonged cold stress), $\mathrm{TP}_{2}$ versus $\mathrm{TP}_{1}$ and $\mathrm{TP}_{4}$ versus $\mathrm{TP}_{3}$ (for recovery and the effect of diurnal fluctuations on metabolite levels) within each variety, and comparisons in every time point between the two varieties (varietal differences). The comparisons for metabolites were presented as $\log _{2}$-transformed of fold change values (Figs. 1, 3; Table S5). A similar approach was also performed for the lipid data (Figs. 2, 4; Table S6). Statistical significance of differences observed between samples 


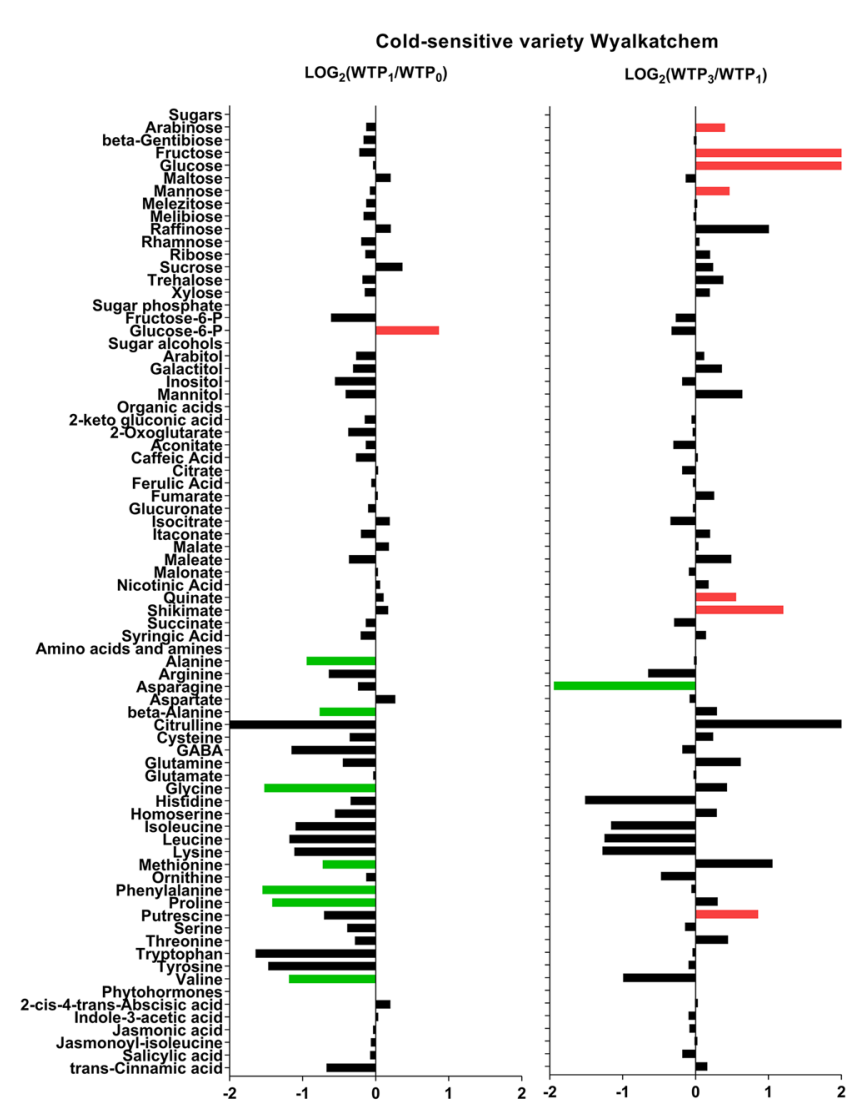

Fig. $1 \log _{2}$-fold changes of primary metabolites in the flag leaves of the cold-sensitive Wyalkatchem (W) and cold-tolerant Young (Y) after one night $\left(\mathrm{TP}_{1}\right.$ vs. $\left.\mathrm{TP}_{0}\right)$ and prolonged $\left(\mathrm{TP}_{3}\right.$ vs. $\left.\mathrm{TP}_{1}\right)$ of cold treatment. Fold changes were calculated by dividing the concentration of the variety at a time point (e.g., $\left.\mathrm{TP}_{1}\right)$ to the concentration of that variety at the previous time point (e.g., $\mathrm{TP}_{0}$ ), then $\log _{2}$-transformed.

was evaluated with the Student's $t$-test in Excel, with a false discovery rate (FDR)-adjusted $p$-value of 0.05 as the cutoff (Benjamini and Hochberg 1995). The statistical analysis for the comparison of the unsaturation to saturation ratio between Wyalkatchem and Young at different time points was also performed with Student's $t$-test using GraphPad Prism 7.0 software (GraphPad Software, La Jolla, CA, USA). All bar plots were created using the same software.

\section{Results}

\subsection{Establishment of a controlled environment assay for chilling tolerance}

To establish a controlled environment phenotyping method for chilling and frost tolerance in wheat, we used two wheat varieties that consistently performed better (Young) and worse (Wyalkatchem) in terms of grain yield in the

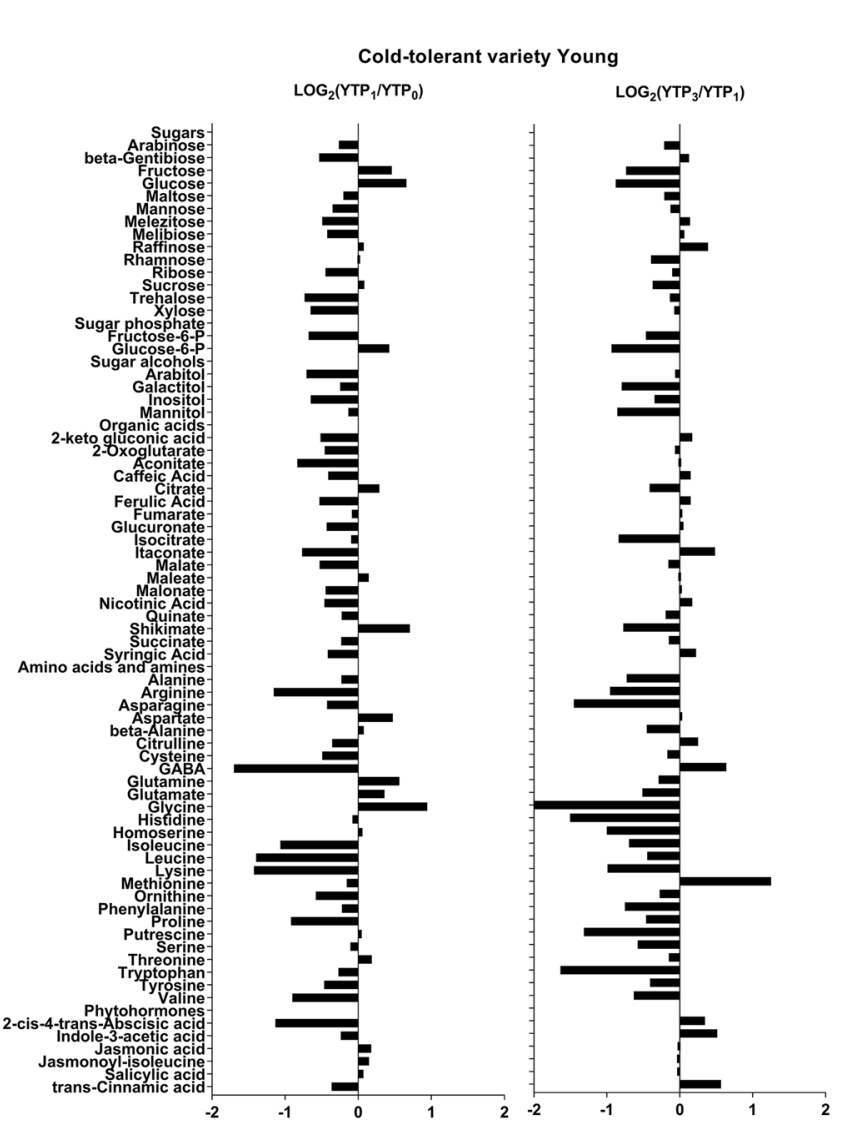

Significance of difference was determined by Benjamini and Hochberg method (Benjamini and Hochberg 1995) with false discovery rate (FDR)-adjusted $p$-value of 0.05 as the cut-off. Green significant decrease, Red significant increase. There were four biological replicates $(n=4)$ for all the measured metabolites, except phytohormones $(\mathrm{n}=3)$

Australian National Frost Initiative (NFI) field trials. We used a linear cooling gradient descending from 21 to $-3{ }^{\circ} \mathrm{C}$ over a period of $4 \mathrm{~h}$, followed by a continuous cold period at $-3{ }^{\circ} \mathrm{C}$ over $8 \mathrm{~h}$ (Fig. S1a). A four-day chilling treatment at the YM stage showed that the average spike grain number relative to unstressed control plants was consistently higher for Young compared to Wyalkatchem, with Wyalkatchem grain number reduced to an average of $76.3 \%$ of control (Fig. S1b). Reduced spike grain number in Wyalkatchem was often associated with the development of larger grains (Fig. S1c). A 4-day cold treatment during different stages of reproductive development showed that sensitivity to chilling in Wyalkatchem was highest at Zadok stages 41 to 47 and from 57 to 65 , which corresponded to the YM stage and anthesis respectively. Spike grain numbers in Young were not significantly affected at both stages (Fig. S1d). A time course experiment shows that spike grain numbers in Wyalkatchem were reduced considerably after three days of treatment, while in Young spike grain numbers were reduced 


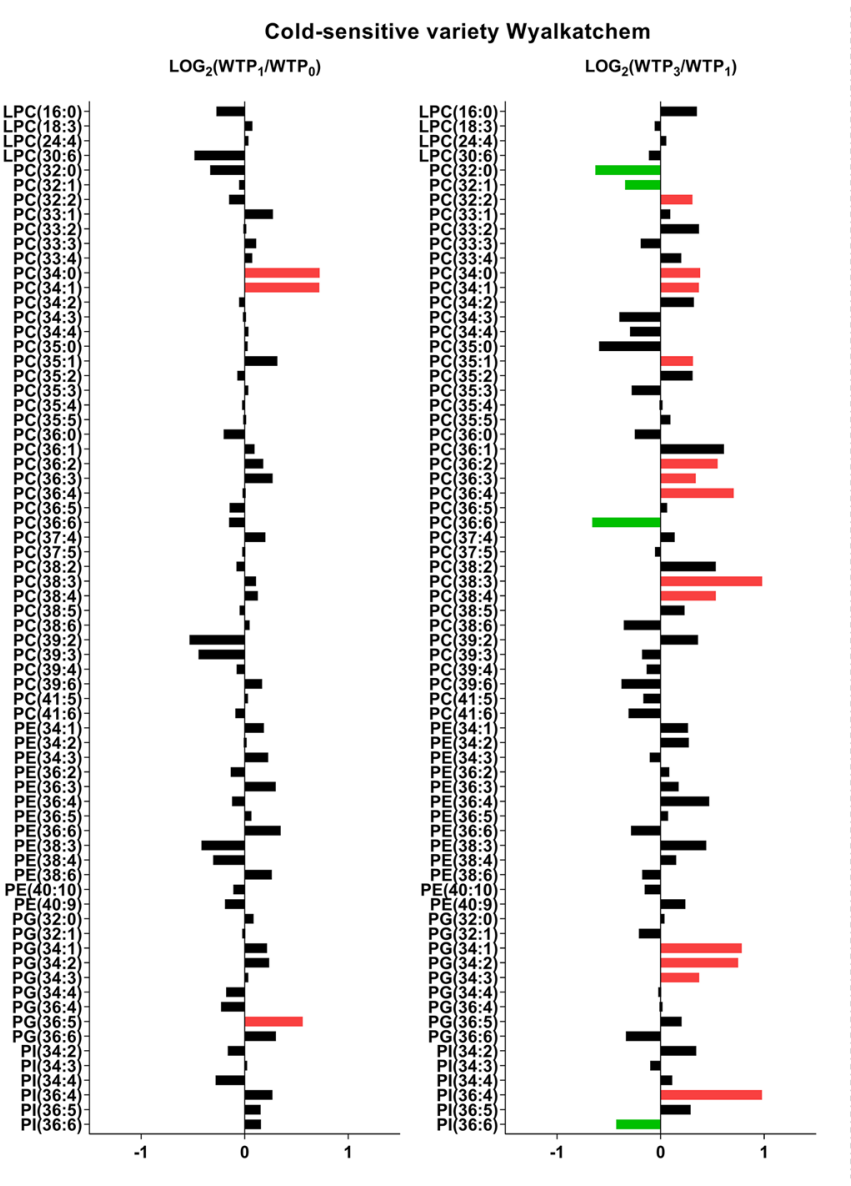

Fig. $2 \log _{2}$-fold changes of phospholipids in the flag leaves of the cold-sensitive Wyalkatchem (W) and cold-tolerant Young (Y) after one night $\left(\mathrm{TP}_{1}\right.$ vs. $\left.\mathrm{TP}_{0}\right)$ and prolonged $\left(\mathrm{TP}_{3}\right.$ vs. $\left.\mathrm{TP}_{1}\right)$ of cold treatment. Fold changes were calculated by dividing the normalized response of the variety at a time point (e.g., $\mathrm{TP}_{1}$ ) to the normalized

significantly from 5 days treatment onwards (Fig. S1e). We therefore standardised on a 4-day YM stage treatment to discriminate the two varieties.

\subsection{Four-day chilling treatment induces significant changes in metabolites and lipids in Young and Wyalkatchem}

To develop a workable field phenotyping method, we focused on cold-induced metabolite changes in the flag leaf, a tissue that is easier to collect in a non-destructive way compared to YM stage spikes. In the field, wheat plants often experience consecutive nights of chilling and frost events throughout flowering, as frost events are linked to large scale climatic patterns and regularly occur over successive nights, while day time temperatures can be normal $\left(\sim 20^{\circ} \mathrm{C}\right)$. Previous chilling events can influence metabolite levels at any harvest time. A time course experiment was therefore designed to identify metabolite changes that were

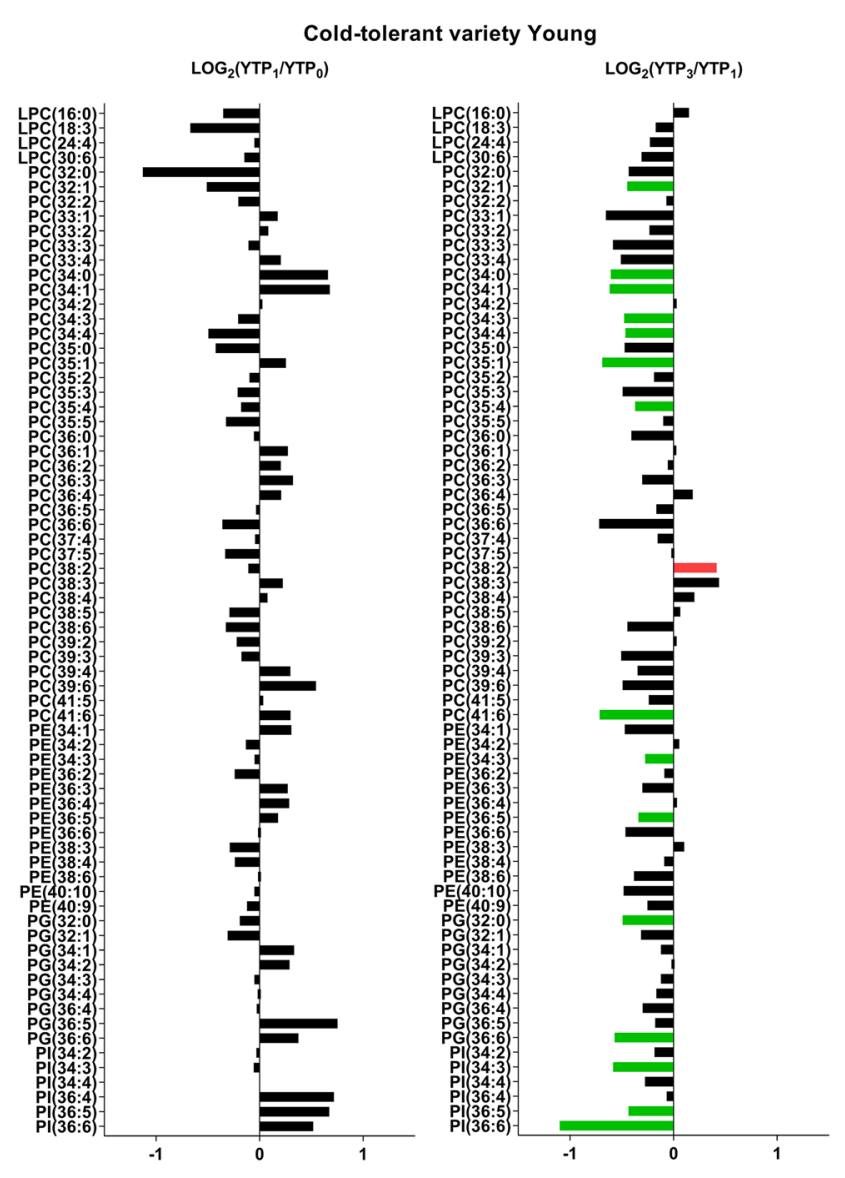

response of that variety at the previous time point (e.g., $\mathrm{TP}_{0}$ ), then $\log _{2}$-transformed. Statistical method and cut-offs are as stated in Fig. 1. Green significant decrease, Red significant increase. There were four biological replicates $(n=4)$ for all the measured lipids

stable over consecutive cold events and were not subject to day-time recovery or to circadian fluctuations. In addition, metabolite markers for chilling tolerance have to accumulate to significantly different levels in the cold-tolerant and sensitive wheat lines.

Using ANOVA multiple comparison analysis and a false discovery rate (FDR)-adjusted $p$-value of 0.05 , significant changes of metabolites and lipids were observed in each variety and between the two varieties across all the time points (Table 1, S7, S8). Cold treatment of cold-sensitive Wyalkatchem revealed significantly more changes in both primary metabolites (25) and lipids (19) compared to coldtolerant Young which showed only one metabolite and ten lipid changes across all the time points (Table 1). When comparing quantitative and qualitative differences in cold acclimation between the two varieties, a total of 43 metabolites and 47 lipids were found to differ across all time points (Table 1). All metabolites and lipids listed in Table 1 were further analysed with Tukey's honestly significant difference 
Table 1 Metabolites and lipids that changed significantly over the 4-day of cold stress in the flag leaves of coldsensitive Wyalkatchem (W) and cold-tolerant Young (Y), analyzed by One-way ANOVA with FDR-adjusted $\mathrm{p}<0.05$ as significance, followed by Tukey Test

Arginine

Asparagine

beta-Alanine

PC(34:0)

Citrate

$\mathrm{PC}(34: 1)$

Citrulline

Fructose

$\operatorname{PC}(34: 3)$

GABA

PC(35:0)

$\mathrm{PC}(35: 1)$

Glucose

Glucose-6-P

Glycine

Histidine

$\mathrm{PC}(36: 2)$

$\mathrm{PC}(36: 3)$

Isoleucine $\quad \mathrm{PC}(36: 4)$

Leucine $\quad$ PC(36:6)

Lysine

$\mathrm{PC}(38: 3)$

Mannose

$\mathrm{PC}(38: 4)$

Phenylalanine

Proline

Putrescine

Shikimate

Sucrose

PG(34:1)

PG(34:2)

Tryptophan $\quad$ PG(34:3)

Tyrosine

Valine

PG(36:5)

Shikimate

$\mathrm{PC}(34: 0)$

PC(34:1)

PC(34:3)

PC(32:0)

$\mathrm{PC}(32: 1)$

\section{2-Oxoglutarate}

Flag leaves_between the two varieties

Wyalkatchem versus Young

Metabolites Lipids

2-cis-4-trans-Abscisic acid

$\operatorname{LPC}(30: 6)$

Aconitate

$\mathrm{PC}(32: 0)$

Alanine

$\mathrm{PC}(32: 1)$

Arabinose

$\mathrm{PC}(32: 2)$

Arabitol

$\mathrm{PC}(33: 1)$

Arginine

$\mathrm{PC}(33: 2)$

Asparagine

$\mathrm{PC}(33: 3)$

beta-Alanine

$\mathrm{PC}(33: 4)$

$\mathrm{PC}(34: 0)$

Citrate

PC(34:1)

Citrulline

$\mathrm{PC}(34: 2)$

Fructose

$\mathrm{PC}(34: 3)$

Fructose-6-P

PC(34:4)

GABA

$\mathrm{PC}(35: 0)$

Galactitol

PC(35:1)

Glucose

$\mathrm{PC}(35: 2)$

$\mathrm{PC}(35: 3)$

Glucose-6-P

$\mathrm{PC}(35: 3)$

Glycine

$\mathrm{PC}(35: 4)$

Histidine

$\mathrm{PC}(35: 5)$

Isoleucine
PG(32:0)

Homoserine

Inositol

Isocitrate

Leucine

Lysine

Maltose

Mannose

Methionine

Phenylalanine

Proline

Putrescine

Quinate

Raffinose

Ribose

Salicylic acid

Serine

Shikimate

Sucrose

trans-Cinnamic acid

Trehalose

Tyrosine

PG(36:5)

Valine

Xylose

PC(36:1)

PC(36:3)

PC(36:4)

PC(36:6)

$\mathrm{PC}(37: 4)$

PC(38:3)

PC(38:4)

PC(38:6)

PC(39:3)

PC(41:6)

PE(34:3)

$\operatorname{PE}(36: 4)$

PE(36:6)

PE(38:3)

PE(38:6)

PE(40:10)

PG(32:0)

PG(32:1)

PG(34:1)

PG(34:2)

PG(34:3)

PG(36:4)

PG(36:5)

PG(36:6)

$\mathrm{PI}(34: 3)$

PI(36:4)

PI(36:5)

PI(36:5)

PI(36:6) 
(HSD) post hoc test in order to determine which pair-wise combinations showed significant differences for both primary metabolites and lipids (Tables S9, S10). Many pairwise combinations were shown to differ significantly for both metabolites (387; highlighted in Table S9) and lipids (470; highlighted in Table S10) for the two wheat varieties and across all time points. But only some of these comparisons are relevant for identification of potential metabolite markers for chilling and frost tolerance phenotyping. Comparisons between $\mathrm{TP}_{1}$ versus $\mathrm{TP}_{0}$ (short cold treatment) and $\mathrm{TP}_{3}$ versus $\mathrm{TP}_{1}$ (stability over prolonged cold treatment during cold acclimation), as well as comparisons between the day and night-time samples $\left(\mathrm{TP}_{2}\right.$ vs. $\mathrm{TP}_{1}$ and $\mathrm{TP}_{4} \mathrm{vs}$. $\mathrm{TP}_{3}$; effect of diurnal fluctuations on metabolite levels), and comparison between these time points for the two wheat varieties are essential to identify those metabolites that could be used as stable markers for cold-tolerance phenotyping. To achieve this particular aim we performed additional Student's $t$-test to carry out pair-wise comparisons to identify candidate metabolite markers that are able to differentiate the response in cold acclimation and cold tolerance between the two wheat varieties.

\subsection{A 4-day chilling time course reveals significant differences in cold acclimation in Wyalkatchem and Young flag leaves}

Sample comparisons: $T P_{1}$ versus $T P_{\alpha} T P_{2}$ versus $T P_{1}, T P_{3}$ versus $T P_{1}$ and $T P_{4}$ versus $T P_{3}$ for both varieties After the first night of cold exposure, the sensitive variety Wyalkatchem showed the most dramatic changes in primary metabolite levels $\left(\mathrm{WTP}_{1}\right.$ vs. $\left.\mathrm{WTP}_{0}\right)$. As shown in Fig. 1 , seven amino acids and amines were significantly decreased, including alanine (-1.9-fold), beta-alanine (-1.7-fold), glycine (-2.9-fold), methionine ( -1.7 -fold), phenylalanine $(-2.9$-fold $)$, proline $(-2.7$-fold $)$ and valine ( -2.3 -fold). One sugar increased significantly at $\mathrm{TP}_{1}$ : glucose-6-phosphate $(+1.8$-fold $)$. There was no significant change in phytohormones. Three lipids increased significantly after overnight cold exposure of Wyalkatchem (Fig. 2): PC(34:0) (+ 1.6-fold), PC(34:1) (+1.6-fold) and PG(36:5) (+1.4-fold). In contrast to Wyalkatchem, the cold tolerant variety Young did not show any significant changes in primary metabolites or lipids at $\mathrm{TP}_{1}$ compared to $\mathrm{TP}_{0}$ (Figs. 1, 2).

To test for recovery and the effect of diurnal fluctuations on metabolite levels we harvested the $\mathrm{TP}_{2}$ samples $6 \mathrm{~h}$ after the $\mathrm{TP}_{1}$ samples, when plants were allowed to experience day-light and normal temperatures $\left(21^{\circ} \mathrm{C}\right)$ for $6 \mathrm{~h}$. Comparison of $\mathrm{TP}_{2}$ and $\mathrm{TP}_{1}$ metabolite levels revealed that some of the significant changes we observed at $\mathrm{TP}_{1}$ in Wyalkatchem flag leaves were partially reversed, but some were maintained and new changes also appeared (Table S5, Fig. S2). Some amino acids and amine levels changed from being decreased at $\mathrm{TP}_{1}$ to being significantly increased at $\mathrm{TP}_{2}$ and regaining $\mathrm{T}_{0}$ levels: citrulline $(+14.9$-fold $)$, glycine $(+2.3$ fold $)$, homoserine $(+2.2$-fold $)$, methionine $(+1.6$-fold $)$ and putrescine $\left(+3.0\right.$-fold). Tryptophan decreased further at $\mathrm{TP}_{2}$ compared to $\mathrm{TP}_{1}$ : (-1.9-fold). There were no differences in phytohormone levels, nor were there any significant changes in lipid levels between $\mathrm{TP}_{2}$ and $\mathrm{TP}_{1}$ Wyalkatchem flag leaves (Table S6, Fig. S3). Comparing $\mathrm{TP}_{2}$ to $\mathrm{TP}_{1}$ in Young showed a significant increase for citrulline $(+2.8$-fold) (Table S5, Fig. S2). There were no significant lipid changes (Table S6, Fig. S3).

After prolonged four-night cold treatment $\left(\mathrm{TP}_{3}\right)$, more significant accumulation of sugars and organic acids were observed in Wyalkatchem flag leaves compared to the first overnight exposure to cold $\left(\mathrm{WTP}_{1}\right.$; Fig. 1). Fructose (+4.1-fold) and glucose (+6.0-fold) increased significantly in Wyalkatchem flag leaves. Also, arabinose $(+1.3$-fold $)$ and mannose $\left(+1.4\right.$-fold) were higher compared to $\mathrm{TP}_{1}$. Two organic acids, quinate $(+1.5$-fold $)$ and shikimate $(+2.3$-fold $)$, and one amine, putrescine $(+1.8$-fold $)$, were significantly higher. One amino acid, asparagine (-3.9fold) was markedly lower at $\mathrm{WTP}_{3}$ compared to $\mathrm{WTP}_{1}$. Prolonged cold treatment of Wyalkatchem also resulted in more lipid changes at $\mathrm{WTP}_{3}$ compared to $\mathrm{WTP}_{1}$. Four lipids were significantly reduced, one being a saturated species $[\mathrm{PC}(32: 0)]$ and the remaining three being unsaturated species [PC(32:1), PC(36:6), PI(36:6)]. Another 13 lipids increased significantly; one of them was a saturated lipid, while 12 others were unsaturated species (Fig. 2, Table S6). In Young, none of the primary metabolites were significantly changed at $\mathrm{YTP}_{3}$ compared to $\mathrm{YTP}_{1}$ (Fig. 1). The most notable changes after prolonged cold stress $\left(\mathrm{YTP}_{3}\right)$ in Young flag leaves compared to the shorter cold stress $\left(\mathrm{YTP}_{1}\right)$ were in the lipid levels. Fifteen lipids were significantly reduced compared to $\mathrm{TP}_{1}$, while one polyunsaturated lipid species, PC(38:2), was significantly higher (1.4-fold). Of the 15 lipids with reduced levels, two were saturated species while a majority (13) were unsaturated species (Fig. 2, Table S6). Comparing $\mathrm{TP}_{4}$ day-time samples to $\mathrm{TP}_{3}$ in Wyalkatchem and Young revealed that there were no significant differences in primary metabolites (Table S5, Fig. S2), while only one polyunsaturated lipid species, PC(41:6) was increased (+ 1.5-fold) in Young flag leaves (Table S6, Fig. S3).

\subsection{Young and Wyalkatchem show significant varietal differences in cold acclimation across time points}

Sample comparisons: WTP versus YTP at each time point In the unstressed plants $\left(\mathrm{TP}_{0}\right)$, there were no significant differences in the levels of primary metabolites or lipids between 


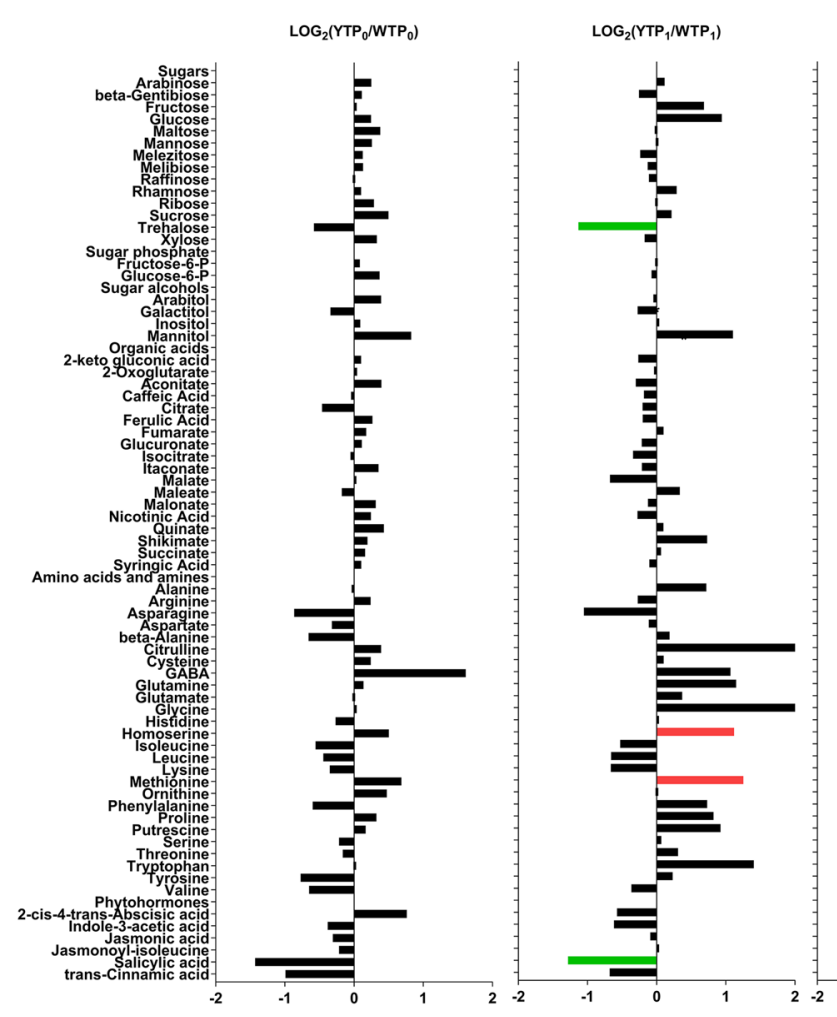

Fig. $3 \log _{2}$-fold changes of primary metabolites in the flag leaves of cold-tolerant Young $(\mathrm{Y})$ compared to the cold-sensitive Wyalkatchem (W) at each time point. Fold changes were calculated by dividing the concentration of $\mathrm{Y}$ to the concentration of $\mathrm{W}$ at that particular time point, then $\log _{2}$-transformed. Statistical method and cut-offs are as

Wyalkatchem and Young flag leaves (Figs. 3, 4). However, several metabolites with potential roles in stress responses were slightly higher in Young compared to Wyalkatchem: sucrose (+1.4-fold), mannitol (+1.8-fold), GABA (+3.1fold), $\mathrm{ABA}\left(+1.7\right.$-fold). At $\mathrm{TP}_{0}$, there were no significant differences between Wyalkatchem and Young in terms of lipid content. However, some lipid levels appeared to be different in Wyalkatchem and Young flag leaves. Some lipids were lower in Young compared to Wyalkatchem when a cutoff of 0.1 was used: PC(33:4) (-1.5-fold), PG(36:6) (-1.6fold) and PI(36:6) (-1.6-fold). Others were higher in Young compared to Wyalkatchem $(p<0.1)$ : $\mathrm{PC}(35: 1)(+1.5$-fold $)$, PG(34:1) (+ 1.4-fold) and PG(34:2) (+1.4-fold) (Fig. 4). Although these lipids (indicated by asterisks) did not satisfy the significance threshold $(p<0.05)$ at $\mathrm{TP}_{0}$, they did become significantly different $(p<0.05)$ after the first night of cold treatment $\left(\mathrm{TP}_{1} ;\right.$ Fig. 4). This may indicate that the lipid composition in Wyalkatchem and Young may be different before cold treatment. This is also reflected by the fact that at $\mathrm{TP}_{0}$, the unsaturated to saturated lipid ratio of Young $(218.6 \pm 10.6)$ was significantly lower than in Wyalkatchem flag leave samples (265.2 \pm 16.0 ; Fig. 5a, Table S11).
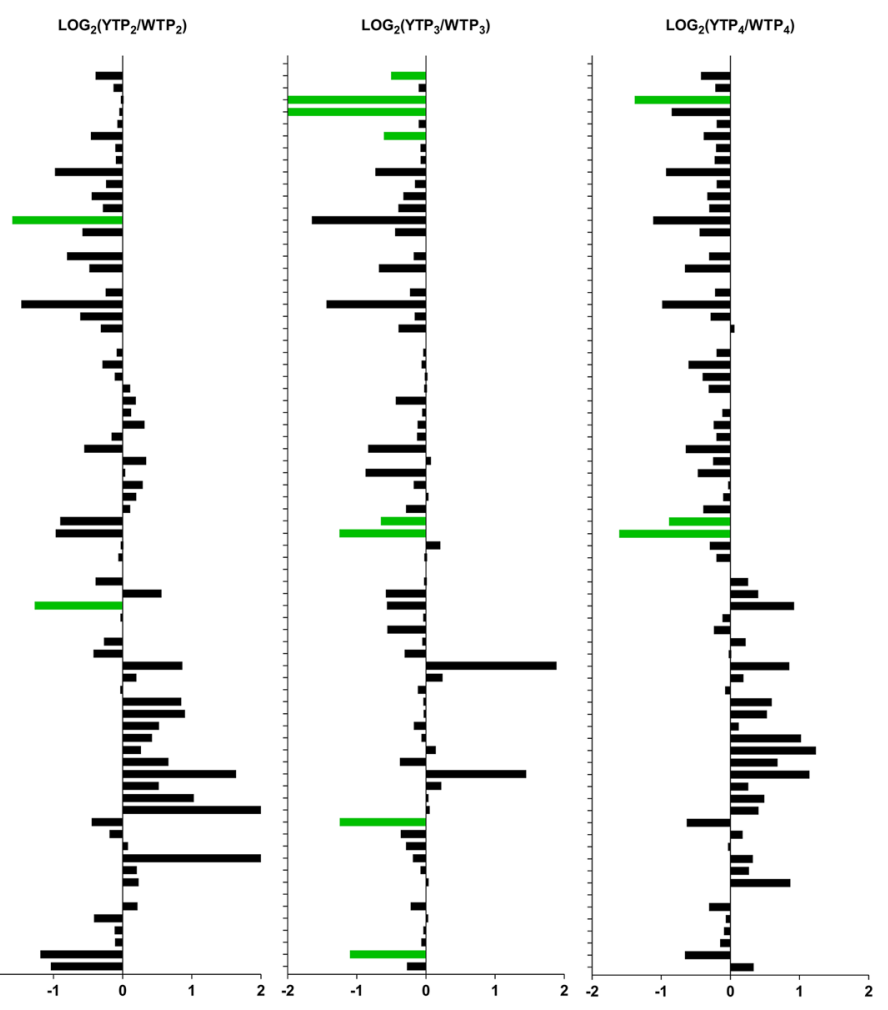

stated in Fig. 1. Green significantly lower in Young/higher in Wyalkatchem; Red significantly higher in Young/lower in Wyalkatchem. There were four biological replicates $(n=4)$ for all the measured primary metabolites, except phytohormones $(n=3)$

After overnight exposure to cold $\left(\mathrm{TP}_{1}\right)$, two amino acids and amines were significantly higher in Young compared to Wyalkatchem (Fig. 3): homoserine (+2.2-fold) and methionine (+2.4-fold). Trehalose (+2.2-fold) was the only sugar that was significantly higher in Wyalkatchem flag leaves. The phytohormone salicylic acid decreased in Young compared to Wyalkatchem (-2.4-fold). Lipids showed the most dramatic difference between the two varieties at $\mathrm{TP}_{1}$. Fourteen lipid species were significantly higher in Young $\mathrm{TP}_{1}$ samples and all were polyunsaturated lipids. Thirteen lipid species were significantly higher in Wyalkatchem compared to Young, and all were also unsaturated lipids (Fig. 4, Table S6). The lipid unsaturation to saturation ratio was not significantly different between Wyalkatchem and Young flag leaves at this time point (Fig. 5a). When comparing the $\mathrm{TP}_{2}$ samples between the two wheat varieties $\left(\mathrm{YTP}_{2}\right.$ vs. $\left.\mathrm{WTP}_{2}\right)$, two metabolites in Young were significantly lower compared to Wyalkatchem (Fig. 3): trehalose (-3.0-fold), and asparagine (-2.4-fold). None of the lipid species were significantly different in Young compared to Wyalkatchem at this time point (Fig. 4, Table S6).There was no significant difference in the lipid unsaturation to saturation ratio between the two varieties at $\mathrm{TP}_{2}$ (Fig. 5a, Table S11). 

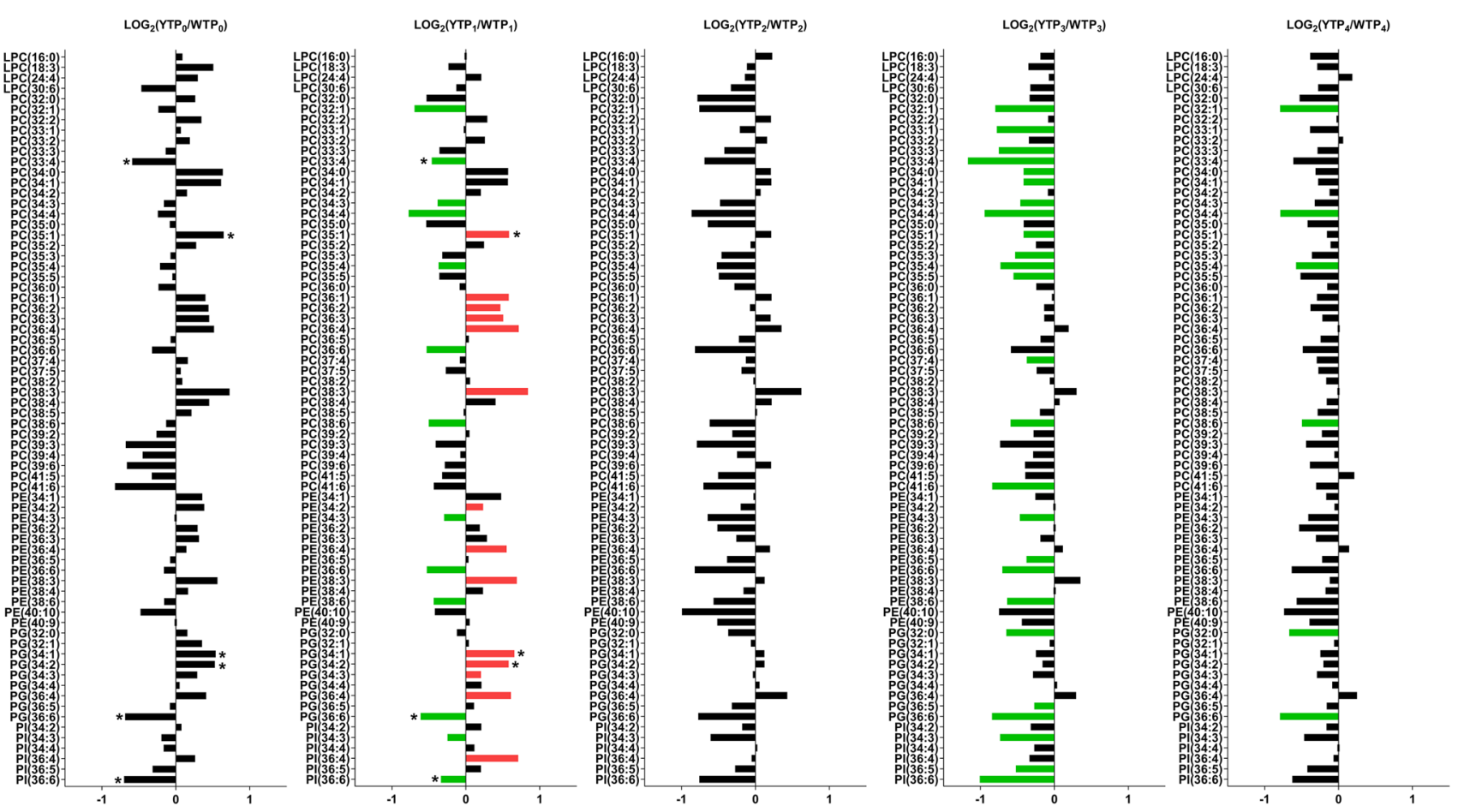

Fig. $4 \log _{2}$-fold changes of phospholipids in the flag leaves of the cold-tolerant Young $(\mathrm{Y})$ compared to the sensitive Wyalkatchem $(\mathrm{W})$ at each time point. Fold changes were calculated by dividing the concentration of $\mathrm{Y}$ to the concentration of $\mathrm{W}$ at that particular time point, then $\log _{2}$-transformed. Statistical method and cut-offs are as stated in Fig. 1. Green significantly lower in Young/higher in Wyalkatchem; Red significantly higher in Young/lower in Wyalkatchem. There were four biological replicates $(n=4)$ for all the measured lipids.
Three lipids: PC(33:4), PG(36:6) and PI(36:6) were lower in Young compared to Wyalkatchem, while another three lipids: $\mathrm{PC}(35: 1)$, PG(34:1) and PG(34:2) were higher in Young compared to Wyalkatchem when a cut-off of 0.1 was used. Although these six lipids (indicated with asterisks at $\mathrm{TP}_{0}$ and $\mathrm{TP}_{1}$ ) did not satisfy the significance threshold $(p<0.05)$ at $\mathrm{TP}_{0}$, they did become significantly different after the first night of cold treatment $\left(\mathrm{TP}_{1}\right)$

unsaturation to saturation ratio of Young was again significantly higher than Wyalkatchem at this stage (Fig. 5a, Table S11).

\subsection{Quantitative changes in two groups of lipids may account for differences in membrane fluidity and cold tolerance between Young and Wyalkatchem}

The time course experiment revealed that the unsaturated to saturated lipid ratio changed progressively from being higher in Wyalkatchem compared to Young at $\mathrm{TP}_{0}$, to higher in Young at $\mathrm{TP}_{3}$ and $\mathrm{TP}_{4}$ (Fig. 5a). This shift in unsaturated to saturated lipid ratio is correlated with differences in the expression profile of two main groups of lipids from $\mathrm{TP}_{0}$ onwards (Fig. 5b, c). The first group of 11 lipids was higher in Wyalkatchem after the first cold exposure $\left(\mathrm{TP}_{1}\right)$ and remained higher than in Young throughout the 4-day time course (Fig. 5b, Table S12). In contrast, another group of 18 lipids (Fig. 5c, Table S13) were on average higher in Young compared to Wyalkatchem from the first exposure to cold 
(a)

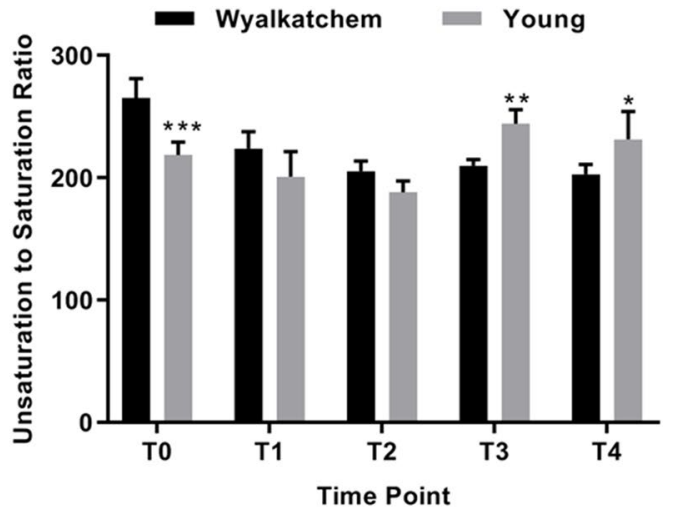

(b)
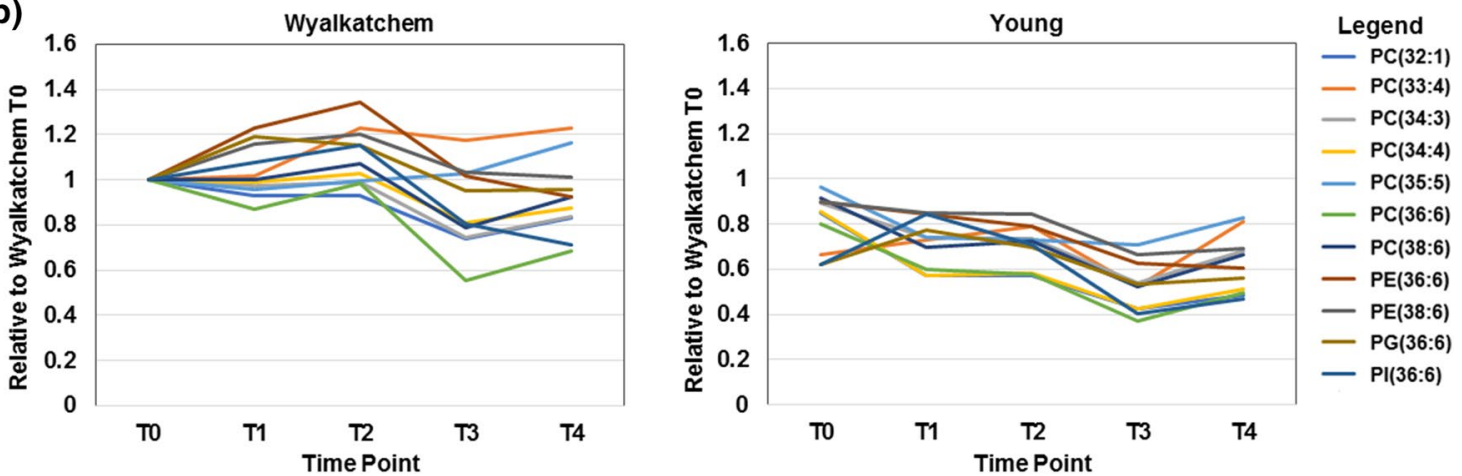

(c)
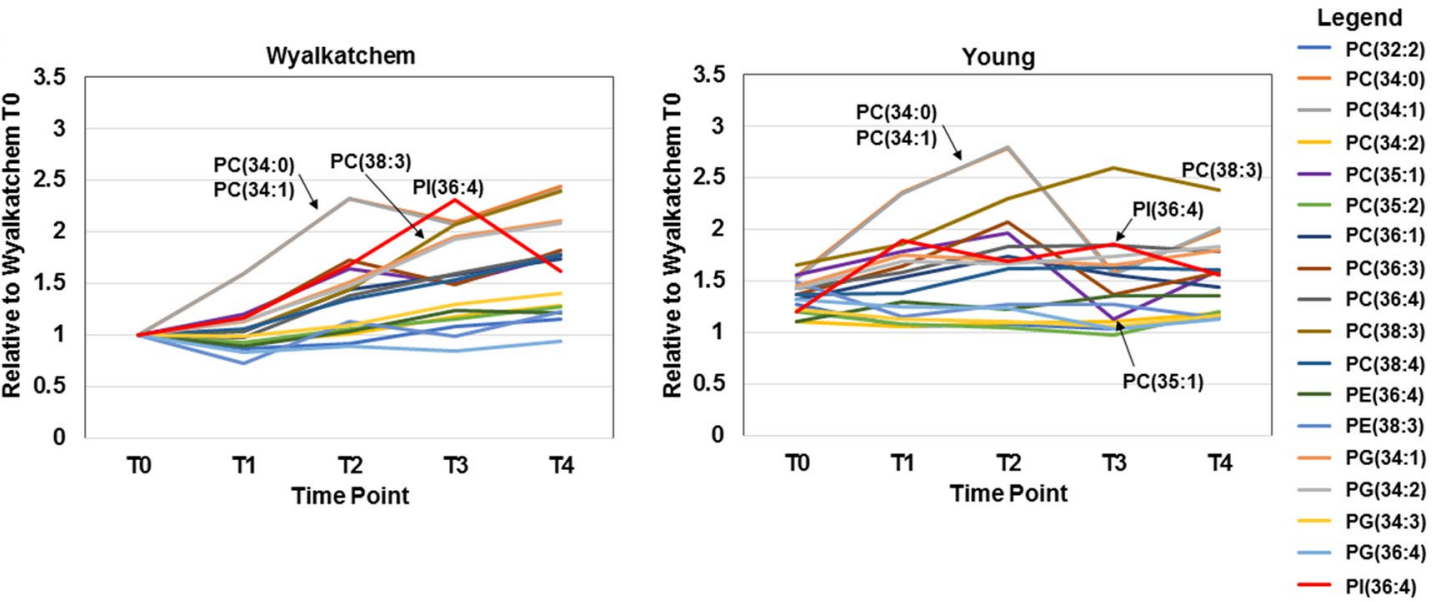

Fig. 5 a Unsaturation to saturation ratio in Wyalkatchem and Young across the time points. Student's t-test was used to compare the ratio values between Wyalkatchem and Young at each time point. Error bars indicate the standard deviation (SD) of four biological replicates $(\mathrm{n}=4)$ and asterisks indicate significance levels: $* \mathrm{p}<0.05,{ }^{*} \mathrm{p}<0.01$ and $* * * \mathrm{p}<0.001$. b Expression levels of a group of 11 lipids with higher expression from $\mathrm{TP}_{1}$ onwards in the cold-sensitive Wyalkatchem compared to Young [(PC(32:1), PC(33:4), PC(34:3), PC(34:4), PC(35:5), PC(36:6), PC(38:6), PE (36:6), PE(38:6), PG(36:6), $\mathrm{PI}(36: 6)]$. These lipids had higher levels in Wyalkatchem after the

onwards $\left(\mathrm{TP}_{1}\right)$, but 5 members of this group behaved quite differently in the two wheat varieties (Fig. 5c). The saturated species PC(34:0), monounsaturated species PC(34:1) and PC (35:1) were significantly increased in both Wyalkatchem

first cold treatment and most of them remained higher than in Young throughout the treatment. c Expression levels of a group of 18 lipids with higher expression from $\mathrm{TP}_{1}$ onwards in the cold-tolerant Young compared to Wyalkatchem [(PC(32:2), PC(34:0), PC(34:1), PC(34:2), PC (35:1), PC(35:2), PC(36:1), PC(36:3), PC(36:4), PC(38:3), PC(38:4), PE(36:4), PE(38:3), PG(34:1), PG(34:2), $\mathrm{PG}(34: 3), \quad P G(36: 4), \quad P I(36: 4)]$. Arrows indicate three lipids, $\mathrm{PC}(34: 0), \mathrm{PC}(34: 1)$ and $\mathrm{PC}(35: 1)$, that changed drastically from $\mathrm{TP}_{2}$ onwards in Young. $\mathbf{b}$ and $\mathbf{c}$ illustrate how these lipids behaved after long term exposure to cold and how they respond to diurnal rhythms

and Young after one overnight cold stress event $\left(\mathrm{TP}_{1}\right)$, but were then reduced drastically in Young compared to Wyalkatchem after the fourth night of cold stress $\left(\mathrm{TP}_{3}\right)$. A fourth lipid, PI(36:4), was increased in Young from time points 
$\mathrm{TP}_{1}$ to $\mathrm{TP}_{3}$, but was induced more strongly in Wyalkatchem after prolonged cold exposure $\left(\mathrm{TP}_{3}\right)$. The unsaturated lipid species PC(38:3) was always significantly higher in Young compared to Wyalkatchem from $\mathrm{TP}_{0}$ onwards and is gradually increased during prolonged cold exposure and was not subjected to diurnal fluctuations $\left(\mathrm{TP}_{2}\right.$ and $\left.\mathrm{TP}_{4}\right) . \mathrm{PC}(38: 3)$ was only increased in Wyalkatchem from $\mathrm{TP}_{3}$ onwards. The combined changes in these two lipid groups and the differential expression behaviour of some members in these lipid groups may contribute towards the observed increase in the ratio of unsaturated to saturated lipids in Young compared to Wyalkatchem (Fig. 5a, Table S11).

\section{Discussion}

\subsection{Wheat chilling and frost tolerance: field versus controlled environment phenotyping}

In the field, phenotyping for wheat frost tolerance is complicated by the unpredictability and spatial/temporal variation in the severity of frost events (Frederiks et al. 2012). Field phenotyping has focused on the presence/absence of tissue damage and yield effects caused by frosts. Frost events at the critical stage of flowering are often catastrophic, leading to the assumption that there is little genetic variation for frost tolerance in wheat (Frederiks et al. 2012; Zheng et al. 2015; Barlow et al. 2015). Non-freezing, chilling conditions occur far more frequently in the field, but the associated sterility and loss in spike grain number has received less attention (Subedi et al. 1998a, b; Chakrabarti et al. 2011; Smith and Zhao 2016). In rice, cold-induced pollen sterility causing loss in grain yield is commonly used as a phenotyping trait (Hayase et al. 1969; Satake et al. 1969; Nishiyama 1984; Oliver et al. 2005). Similar to drought and heat stress, phenotyping for chilling and frost tolerance in the field is compromised by avoidance or escape mechanisms such as phenology of flowering (Fleury et al. 2010; Richards et al. 2010; Shavrukov et al. 2017). Controlled environment phenotyping can overcome some of the pitfalls associated with field work. Occurrence and severity of cold events can be monitored and repeated consistently, as well as their timing during reproductive growth controlled, making it possible to study the physiological and molecular basis of cold tolerance without the interference of avoidance or escape mechanisms or the complexity of changes in frost severity and duration between events. We established a controlled environment screening method based on maintenance of grain number under chilling conditions using two wheat cultivars that were reproducibly shown to be more tolerant (Young) and sensitive (Wyalkatchem) to field chilling and frost conditions. This screening method allowed us to reproduce the field rankings for the two cultivars, as well as the cold tolerance rankings for several other tolerant and sensitive wheat cultivars from the National Frost Initiative (Dolferus et al., unpublished results). Chilling conditions led to a reduction in spike grain number in wheat and we show that the YM and anthesis stages are the two most sensitive stages to chilling stress.

\subsection{Metabolomics as alternative phenotyping tool for chilling and frost tolerance in wheat}

Tolerance to frost requires the establishment of an acclimation response in plant tissues. Cold acclimation starts below a certain non-freezing threshold temperature before belowzero temperatures cause ice formation and desiccation and freezing damage to plant tissues (Livingston et al. 2016). It remains unclear how much time it takes for effective cold acclimation to reach maximal potential, nor do we know whether there is genetic variation in quantitative or qualitative aspects of cold acclimation (Pagter and Arora 2013; Vanková et al. 2014; Chen et al. 2014; Fiebelkorn and Rahman 2016). Understanding what acclimation to non-freezing or chilling conditions involves is essential to understand how frost tolerance (cold, chilling/dessication and freezing) can be improved.

Acclimation to abiotic stresses such as cold leads to accumulation of metabolites in plant tissues to protect cellular functions (Morgan 1992; Ruelland et al. 2009; Ouellet and Charron 2013; Miura and Furumoto 2013). Some of these compounds act as osmoprotectants to protect against abiotic stresses that affect the water balance of plants (Vágújfalvi et al. 1999; Uemura et al. 2003; Chen et al. 2014). Osmolytes are small, electrically neutral, water-soluble organic compounds that efficiently maintain osmotic balance and stabilize membranes and macromolecules under water stress conditions. They include betaines, amino acids, polyols and non-reducing sugars (Burg and Ferraris 2008; Slama et al. 2015; Nahar et al. 2016; Argiolas et al. 2016). Osmolytes also protect membranes and act as scavengers for toxic reactive oxygen species. They are induced by a variety of other abiotic stresses that affect the water balance (drought, heat, salinity), making them therefore less specific markers for cold tolerance per se (Beck et al. 2007). There is no clear evidence available as to how quantitative or qualitative differences in accumulation of these compounds directly contribute to cold tolerance. We compared changes in the concentrations of metabolites and lipids during cold acclimation for two wheat varieties that differ in cold tolerance. Many metabolites are subject to day-night circadian fluctuations and cold or frost events usually occur during the night. It has been demonstrated that there is a substantial overlap between cold and circadian-regulated genes, suggesting that cold acclimation is tightly linked to circadian rhythms (Espinoza et al. 2010; Sanchez et al. 2011). However, it is 
expected that some compounds that accumulate after an initial chilling or frost event during the night remain present in plant tissues as a protection against subsequent chilling events. To investigate this, we designed an experiment where we compared metabolite levels in leaves harvested in the morning immediately after a chilling event and $6 \mathrm{~h}$ later, when plants were allowed to recover in the light at normal temperatures. We also compared these samples for a single and four consecutive chilling cycles. Because our focus is on using metabolites as diagnostic markers for cold acclimation, we first used the easy-to-collect flag leaves.

\subsection{Effect of prolonged/consecutive chilling treatments on wheat flag leaf metabolites and lipids}

Cumulative changes with respect to $\mathrm{TP}_{0}, \mathrm{TP}_{1}$ and $\mathrm{TP}_{3}$ for both Wyalkatchem and Young flag leaves show significant changes occurring after the first cold night $\left(\mathrm{TP}_{1}\right)$, with additional changes appearing after prolonged cold treatment $\left(\mathrm{TP}_{3}\right)$. However, fewer changes were observed in the daytime samples $\left(\mathrm{TP}_{2}\right.$ and $\left.\mathrm{TP}_{4}\right)$, suggesting that partial recovery takes place during normal day temperatures for some metabolites. Day-night fluctuation in levels of some metabolite is likely due to circadian rhythms and fluctuation of photosynthetic activity and associated metabolism and needs to be taken into account for field sampling and cold tolerance phenotyping. After the first night of cold treatment, a significant reduction in the levels of most amino acids and amines was evident in Wyalkatchem, but not in Young. Most amino acids and amines were higher in Young compared to Wyalkatchem from the first overnight cold treatment $\left(\mathrm{TP}_{1}\right)$. Interestingly, the amino acids proline and citrulline, which are known to accumulate in plant tissues under a variety of stresses that affect water relations (drought, salinity, cold, heat; Mayer et al. 1990; Ashraf and Foolad 2007; Lehmann et al. 2010; Hayat et al. 2012; Liang et al. 2013), behaved quite differently in Wyalkatchem compared to Young. Proline was found to decrease significantly after one night cold treatment in Wyalkatchem but increased in Young flag leaves. Citrulline behaved in a similar fashion, but was only found to change significantly at a more relaxed cut-off of $(p<0.1)$ in both wheat lines. Citrulline has hydroxyl radical scavenging and antioxidant properties and can protect DNA and enzymes from oxidative injuries (Kawasaki et al. 2000; Akashi et al. 2001; Rimando and Perkins-Veazie 2005; Kusvuran et al. 2013).

In Young, a significant increase in citrulline was observed in the $\mathrm{TP}_{2}$ samples. GABA, an amine with cryo-protective properties (Bouche and Fromm 2004; Mazzucotelli et al. 2006) was also marginally increased-albeit below significance levels. After the prolonged cold treatment $\left(\mathrm{TP}_{3}\right)$, amino acid levels in Wyalkatchem did not fully recover and citrulline increased further. Accumulation of citrulline occurred predominantly in Wyalkatchem and only temporarily in Young $\left(\mathrm{TP}_{1}\right)$. Wyalkatchem also showed a threefold increase for the polyamine putrescine, an amino acid breakdown product with growth-regulatory properties known to play a role in abiotic stresses (Gill and Tuteja 2010; Shi and Chan 2014). Shikimate, a substrate for the synthesis of lignin and various aromatic compounds such as quinate (Herrmann and Weaver 1999; Guo et al. 2014) was increased in Wyalkatchem but decreased in Young. The enzymes of this pathway have been shown to be involved in defence responses (Kasai et al. 2005). Furthermore, compatible osmolytes such as sugars (arabinose, glucose, glucose6-P,fructose and raffinose) and the sugar alcohol mannitol also accumulated in Wyalkatchem upon cold treatment, but not in Young flag leaves. Sugars and sugar alcohols play a role as osmo-and cryo-protectants (Loescher et al. 1992; Travert et al. 1997; Burg and Ferraris 2008; Slama et al. 2015). The accumulation of these osmolytes indicates that Wyalkatchem is responding to water or desiccation stress as a consequence of cold treatment. Repression of amino acid synthesis in Wyalkatchem may also contribute to this situation. None of these symptoms was observed in tolerant variety Young. Osmolytes can therefore serve as markers for chilling sensitivity; they may be indicative of water stress as a result of membrane damage caused by chilling and frost. But osmolytes point to a secondary effect of cold treatment and their accumulation is therefore not specific for low temperature stress. Osmolyte accumulation can be activated by other abiotic stresses as well (drought, heat, salinity; Beck et al. 2007). We did not observe many significant differences in phytohormone levels between Wyalkatchem and Young. Interestingly, the only phytohormone that showed significant differences between the two wheat varieties was salicylic acid. Trans-cinnamic acid (significant at $\mathrm{TP}_{2}$ ), one of the precursors of salicylic acid biosynthesis (Hayat et al. 2007), follows the same abundance profile as salicylic acid. Salicylic acid levels were consistently higher in Wyalkatchem compared to Young at both short and prolonged cold stress exposures. Salicylic acid is known for its role in plant pathogen responses, but also plays a role in abiotic stress in regulating stomatal closure under water stress. When applied to plants, salicylic acid was shown to reduce freezing tolerance and at high levels causes oxidative stress and increased stress sensitivity (Lissarre et al. 2010; Miura and Tada 2014; Eremina et al. 2016).

Cold treatment of Wyalkatchem and Young flag leaves resulted in quantitative and qualitative changes in the lipid composition and the lipid changes outnumber those observed for the primary metabolites. The most prevalent changes in cold-stressed Wyalkatchem and Young flag leaves were 
observed for the phospatidylcholine (PC) class of lipids. PCs are the most abundant plasma membrane lipids in plants and animals. Differences between Wyalkatchem and Young PC levels in response to cold may therefore contribute to changes in plasma membrane fluidity and permeability (Upchurch 2008; van Meer et al. 2008). The saturated lipid species PC(34:0), monounsaturated species PC(34:1) and $\mathrm{PC}(35: 1)$ increased in both wheat varieties after the first exposure to cold $\left(\mathrm{TP}_{1}\right)$ and then decreased after prolonged cold treatment $\left(\mathrm{TP}_{3}\right)$ (Fig. 5c). However, the decrease of these $\mathrm{PCs}$ at $\mathrm{TP}_{3}$ was faster in Young compared to Wyalkatchem and this may have contributed to a higher level of unsaturated lipids in Young compared to Wyalkatchem (Fig. 5c). Even at $\mathrm{TP}_{0}$, Young and Wyalketchem flag leaves show a different lipid saturation:unsaturation ratio and some lipid levels are marginally different, but become significantly different after the first night of cold treatment. This seems to indicate that Young is better adapted to cold from the beginning. The change in the unsaturated to saturated lipid ratio is the result of a change in relatively few lipid species and this may be the result of a modification in the expression of a small number of genes. The fact that Young and Wyalkatchem show a different lipid content before and after cold treatment may indicate that both lines are adapted differently to cold temperatures and this may be due to genetic variation in the regulation or function of lipid biosynthesis gene/genes. Using lipids as phenotyping markers may enable us to identify those genes using a mapping population. The superior ability of the tolerant variety Young to adjust and remodel its membrane composition may be the key factor to overcome prolonged cold exposure. We do not know to what extend the differences in lipid content between Young and Wyalkatchem flag leaves can protect against frost (temperature threshold) or whether the observed differences are sufficient to survive more severe frost conditions. But using lipidomics to screen wheat germplasm collections for varieties with higher lipid unsaturation levels than Young may hold the key to improving frost tolerance in wheat. Plants produce a large variety of lipid species in their chloroplasts and the fact that the spectrum of synthesised lipids depends significantly on the reigning environmental conditions (e.g., temperature) indicates that modification of membrane lipid composition is an important environmental adaption mechanism (Aid 2019).

Phosphatidylglycerol lipids (PGs) are the main phospholipid class of thylakoid membranes in higher plants (Ren et al. 2014). PGs are the second largest lipid group that is affected by cold in wheat flag leaves. The saturated lipid PG(32:0) is lower in Young and higher in Wyalkatchem flag leaves after the prolonged cold stress $\left(\mathrm{TP}_{3}\right)$, indicating that this lipid species may also contribute to the higher degree of lipid unsaturation in membranes of the tolerant variety Young. Phosphotidylinositol (PI) lipids are the third most abundant extraplastidic lipid. PIs are involved in membrane signalling under abiotic stress conditions such as osmotic stress (Munnik and Vermeer 2010; Zheng et al. 2016). PIs increase by $50 \%$ after freezing in Arabidopsis and contribute to frost tolerance (Zheng et al. 2016). Significantly higher levels in Young compared to Wyalkatchem of polyunsaturated PI species such $\mathrm{PI}(36: 4)$ at $\mathrm{TP}_{1}$ may also contribute to better membrane protection for the tolerant variety during cold stress.

Plants respond with complex changes in lipid composition during cold acclimation to maintain membrane integrity (Uemura and Steponkus 1994; Uemura et al. 1995; Degenkolbe et al. 2012). The amount of double bonds in unsaturated lipids improves membrane fluidity at lower temperatures. To maintain optimum membrane fluidity, unsaturation levels of lipids decreases at higher temperatures and increases at lower temperatures (Narayanan et al. 2016a, b; Zheng et al. 2016). The significant increase in the ratio of unsaturated to saturated lipids after prolonged cold treatment suggests that Young is better able to adapt its membrane composition to low temperatures. Failure of Wyalkatchem to modify lipid composition in favour of unsaturated to saturated lipids may lead to membrane damage, causing electrolyte leakage and water loss, leading to the osmotic stress response observed in this variety. The lipid changes we observed in Young and Wyalkatchem flag leaves are part of an acclimation response to cold; they are amplified during prolonged cold treatment, they are not subjected to diurnal fluctuations and they are specific for low temperature adaptation. Lipids are therefore suitable markers for chilling and frost tolerance phenotyping.

\section{Conclusion}

In conclusion, combined metabolome and lipidome analyses in flag leaves have shed light on important differences in cold acclimation between the cold-sensitive Wyalkatchem and cold-tolerant Young varieties. The main differences between the two wheat cultivars were the differential modification of membrane lipids in both varieties and the establishment of an osmotic stress-like response in the sensitive varieties Wyalkatchem. There is a gradual change in the ratio of unsaturated versus saturated lipids that favors higher levels of unsaturated lipids being incorporated in the tolerant variety Young. As a consequence, Young may acquire a superior ability to adapt membrane fluidity to cold conditions and avoid membrane damage. Diagnostic metabolites, including sugars and lipids, can therefore serve as tools to improve the reliability of phenotyping for chilling and frost tolerance in wheat. Furthermore, metabolomics and lipidomics can be combined with transcriptome analysis and genomics approaches to identify the genetic mechanism driving the 
observed differences in metabolite accumulation and this could lead to future DNA marker-based approaches for frost tolerance breeding in wheat.

Acknowledgements Cold treatments were carried out using growth chambers from the High- Resolution Plant Phenomics Centre in Canberra (HRPPC; https://www.plantphenomics.org.au/tag/high-resolution -plant-phenomics-centre/). The authors thank Mrs Cheka Kehelpannala from the School of Biosciences, Mrs Nirupama Jayasinghe, Mrs Himasha Mendis, Ms. Veronica Lui and Dr Siria Natera from Metabolomics Australia for the metabolite, lipid and phytohormone analyses. Metabolomics Australia (School of BioSciences, The University of Melbourne, Australia) is a National Collaborative Research Infrastructure Strategy initiative under Bioplatforms Australia Pty Ltd (http://www.bioplatfor ms.com/).

Author contributions BEC performed part of the metabolite, lipid and phytohormone experiments and analysed the data. WWHH and TWTR contributed to the data analysis. UR designed the metabolite analysis and contributed to data analysis, statistical analysis and manuscript writing. RD conceived the project, designed the experiments and contributed to the writing of the manuscript. RD, XW, and TR established the controlled environmental conditions for chilling treatments and harvested the plant tissue samples for metabolite analysis. BB provided advice on the wheat germplasm to best use in this study and provided field climatic information, as well as advice to establish the controlled environmental conditions most likely to provide discriminating damage based on field experience in phenotyping the same material. BEC, WWHH, TWTR and RD wrote the manuscript.

Funding This research was supported by Grants of the Australian Grains Research and Development Corporation (GRDC projects CSP00143 and CSP00202).

Data availability statement All the data sets that support the findings of this study are available as Supplemental data files (Microsoft Excel 2013 files).

\section{Compliance with ethical standards}

Conflict of interest The authors declare that they have no conflict of interest.

Research involved human and animal participants This article does not contain any studies with human and/or animal participants performed by any of the authors.

Open Access This article is distributed under the terms of the Creative Commons Attribution 4.0 International License (http://creativeco mmons.org/licenses/by/4.0/), which permits unrestricted use, distribution, and reproduction in any medium, provided you give appropriate credit to the original author(s) and the source, provide a link to the Creative Commons license, and indicate if changes were made.

\section{References}

Aid, F. (2019). Plant lipid metabolism. In R. V. Baez (Ed.), Lipid metabolism. Vienna: IntechOpen.

Akashi, K., Miyake, C., \& Yokota, A. (2001). Citrulline, a novel compatible solute in drought-tolerant wild watermelon leaves, is an efficient hydroxyl radical scavenger. FEBS Letters, 508(3), 438-442.

Argiolas, A., Puleo, G. L., Sinibaldi, E., \& Mazzolai, B. (2016). Osmolyte cooperation affects turgor dynamics in plants. Scientific Reports, 6, 30139.

Ashraf, M., \& Foolad, M. R. (2007). Roles of glycine betaine and proline in improving plant abiotic stress resistance. Environmental and Experimental Botany, 59(2), 206-216.

Barlow, K. M., Christy, B. P., O’Leary, G. J., Riffkin, P. A., \& Nuttall, J. G. (2015). Simulating the impact of extreme heat and frost events on wheat crop production: A review. Field Crops Research, 171, 109-119.

Beck, E. H., Fettig, S., Knake, C., Hartig, K., \& Bhattarai, T. (2007). Specific and unspecific responses of plants to cold and drought stress. Journal of Biosciences, 32(3), 501-510.

Benjamini, Y., \& Hochberg, Y. (1995). Controlling the false discovery rate: A practical and powerful approach to multiple testing. Journal of the Royal Statistical Society (Series B), 57(1), 289-300.

Bohn, M., Luthje, S., Sperling, P., Heinz, E., \& Dorffling, K. (2007). Plasma membrane lipid alterations induced by cold acclimation and abscisic acid treatment of winter wheat seedlings differing in frost resistance. Journal of Plant Physiology, 164(2), 146-156.

Bouche, N., \& Fromm, H. (2004). GABA in plants: Just a metabolite? Trends in Plant Science, 9(3), 110-115.

Boughton, B. B., Callahan, D. L., Silva, C., Bowne, J., Nahid, A., Rupasinghe, T., et al. (2011). Comprehensive profiling and quantitation of amine group containing metabolites. Analytical Chemistry, 83, 7523-7530.

Burg, M. B., \& Ferraris, J. D. (2008). Intracellular organic osmolytes: Function and regulation. Journal of Biological Chemistry, 283(12), 7309-7313.

Cao, D., Lutz, A., Hill, C. B., Callahan, D. L., \& Roessner, U. (2017). A quantitative profiling method of phytohormones and other metabolites applied to barley roots subjected to salinity stress. Frontiers in Plant Science, 7, 2070.

Chakrabarti, B., Singh, S. D., Nagarajan, S., \& Aggarwal, P. K. (2011). Impact of temperature on phenology and pollen sterility of wheat varieties. Australian Journal of Crop Science, 5(8), 1039-1043.

Chen, Y., Jiang, J., Chang, Q., Gu, C., Song, A., Chen, S., et al. (2014). Cold acclimation induces freezing tolerance via antioxidative enzymes, proline metabolism and gene expression changes in two Chrysanthemum species. Molecular Biology Reports, 41(2), 815-822.

Crimp, S., Bakar, K. S., Kokic, P., Jin, H., Nicholls, N., \& Howden, M. (2015). Bayesian space-time model to analyse frost risk for agriculture in Southeast Australia. International Journal of Climatology, 35(8), 2092-2108.

Degenkolbe, T., Giavalisco, P., Zuther, E., Seiwert, B., Hincha, D. K., \& Willmitzer, L. (2012). Differential remodeling of the lipidome during cold acclimation in natural accessions of Arabidopsis thaliana. Plant Journal, 72(6), 972-982.

Demotes-Mainard, S., Doussinault, G., Meynard, J. M., \& Gate, P. (1996). Is it possible to diagnose at harvest a problem of pollen sterility in wheat? European Journal of Agronomy, 5(3-4), 169-180.

Dias, D. A., Hill, C. B., Jayasinghe, N. S., Atieno, J., Sutton, T., \& Roessner, U. (2015). Quantitative profiling of polar primary metabolites of two chickpea cultivars with constrasting responses to salinity. Journal of Chromatography B, 1000, 1-13.

Dolferus, R., Powell, N., Ji, X., Ravash, R., Edlington, J., Oliver, S., et al. (2013). The physiology of reproductive-stage abiotic stress tolerance in cereals. In G. Rout \& A. Das (Eds.), Molecular stress physiology of plants (pp. 193-216). India: Springer.

Douglas, D. J. (2009). Linear quadruples in mass spectrometry. Mass Spectrometry Reviews, 28, 937-960. 
Eremina, M., Rozhon, W., \& Poppenberger, B. (2016). Hormonal control of cold stress responses in plants. Cellular and Molecular Life Sciences, 73(4), 797-810.

Espinoza, C., Degenkolbe, T., Caldana, C., Zuther, E., Leisse, A., Willmitzer, L., et al. (2010). Interaction with diurnal and circadian regulation results in dynamic metabolic and transcriptional changes during cold acclimation in Arabidopsis. PLoS ONE, 5(11), e14101.

Fiebelkorn, D., \& Rahman, M. (2016). Development of a protocol for frost-tolerance evaluation in rapeseed/canola (Brassica napus $\mathrm{L}$ ). Crop Journal, 4(2), 147-152.

Fleury, D., Jefferies, S., Kuchel, H., \& Langridge, P. (2010). Genetic and genomic tools to improve drought tolerance in wheat. Journal of Experimental Botany, 61(12), 3211-3222.

Folch, J., Lees, M., \& Stanley, G. H. S. (1957). A simple method for the isolation and purification of total lipids from animal tissues. Journal of Biological Chemistry, 226, 497-509.

Fowler, D. B., Breton, G., Limin, A. E., Mahfoozi, S., \& Sarhan, F. (2001). Photoperiod and temperature interactions regulate lowtemperature-induced gene expression in barley. Plant Physiology, 127(4), 1676-1681.

Frederiks, T. M., Christopher, J. T., Fletcher, S. E. H., \& Borrell, A. K. (2011). Post head-emergence frost resistance of barley genotypes in the northern grain region of Australia. Crop and Pasture Science, 62(9), 736-745.

Frederiks, T. M., Christopher, J. T., Harvey, G. L., Sutherland, M. W., \& Borrell, A. K. (2012). Current and emerging screening methods to identify post-head-emergence frost adaptation in wheat and barley. Journal of Experimental Botany, 63(15), 5405-5416.

Gill, S. S., \& Tuteja, N. (2010). Polyamines and abiotic stress tolerance in plants. Plant Signaling \& Behavior, 5, 26-33.

Gray, G. R., Chauvin, L.-P., Sarhan, F., \& Huner, N. P. (1997). Cold acclimation and freezing tolerance (a complex interaction of light and temperature). Plant Physiology, 114, 467-474.

Guo, J., Carrington, Y., Alber, A., \& Ehlting, J. (2014). Molecular characterization of quinate and shikimate metabolism in Populus trichocarpa. Journal of Biological Chemistry, 289, 23846-23858.

Guy, C. L. (1990). Cold acclimation and freezing stress tolerance: Role of protein metabolism. Annual Review of Plant Physiology and Plant Molecular Biology, 41, 187-223.

Guy, C. (1999). Molecular responses of plants to cold shock and cold acclimation. Journal of Molecular Microbiology Biotechnology, 1(2), 231-242.

Hayase, H., Satake, T., Nashiyama, I., \& Ito, N. (1969). Male sterility caused by cooling treatment at the meiotic stage in rice plants. II. The most sensitive stage to cooling and fertilizing ability of pistils. Proceedings of the Crop Science. Society of Japan, 38, 706-711.

Hayat, S., Ali, B., \& Ahmad, A. (2007). Salicylic acid: Biosynthesis, metabolism and physiological role in plants. In S. Hayat \& A. Ahmad (Eds.), Salicylic acid: A plant hormone (pp. 1-14). Dordrecht: Springer.

Hayat, S., Hayat, Q., Alyemeni, M. N., Wani, A. S., Pichtel, J., \& Ahmad, A. (2012). Role of proline under changing environments: A review. Plant Signaling \& Behavior, 7(11), 1456-1466.

Herrmann, K. M., \& Weaver, L. M. (1999). The shikimate pathway. Annual Review of Plant Physiology and Plant Molecular Biology, 50, 473-503.

Horvath, E., Szalai, G., \& Janda, T. (2007). Induction of abiotic stress tolerance by salicylic acid signaling. Journal of Plant Growth Regulation, 26(3), 290-300.

Janmohammadi, M. (2012). Metabolomic analysis of low temperature responses in plants. Current Opinion in Agriculture, 1, 1-6.

Javadian, N., Karimzadeh, G., Mahfoozi, S., \& Ghanati, F. (2010). Cold-induced changes of enzymes, proline, carbohydrates, and chlorophyll in wheat. Russian Journal of Plant Physiology, 57(4), 540-547.

Ji, X., Shiran, B., Wan, J., Lewis, D. C., Jenkins, C. L. D., Condon, A. G., et al. (2010). Importance of pre-anthesis anther sink strength for maintenance of grain number during reproductive stage water stress in wheat. Plant, Cell and Environment, 33(6), 926-942.

Jorge, T. F., Rodrigues, J. A., Caldana, C., Schmist, R., van Dongen, J. T., Thomas-Oates, J., et al. (2016). Mass spectrometry-based plant metabolomics: Metabolite responses to abiotic stress. Mass Spectrometry Reviews, 35, 620-649.

Kalberer, S. R., Wisniewski, M., \& Arora, R. (2006). Deacclimation and reacclimation of cold-hardy plants: Current understanding and emerging concepts. Plant Science, 171(1), 3-16.

Kasai, K., Kanno, T., Akita, M., Ikejiri-Kanno, Y., Wakasa, K., \& Tozawa, Y. (2005). Identification of three shikimate kinase genes in rice: characterization of their differential expression during panicle development and of the enzymatic activities of the encoded proteins. Planta, 222(3), 438-447.

Kawasaki, S., Miyake, C., Kohchi, T., Fujii, S., Uchida, M., \& Yokota, A. (2000). Responses of wild watermelon to drought stress: Accumulation of an ArgE homologue and citrulline in leaves during water deficits. Plant and Cell Physiology, 41(7), 864-873.

Kurepin, L. V., Dahal, K. P., Savitch, L. V., Singh, J., Bode, R., Ivanov, A. G., et al. (2013). Role of CBFs as integrators of chloroplast redox, phytochrome and plant hormone signaling during cold acclimation. International Journal of Molecular Sciences, 14(6), 12729-12763.

Kusvuran, S., Dasgan, H. Y., \& Abak, K. (2013). Citrulline is an important biochemical indicator in tolerance to saline and drought stresses in melon. The Scientific World Journal, 2013, 253414.

Leff, B., Ramankutty, N., \& Foley, J. A. (2004). Geographic distribution of major crops across the world. Global Biogeochemical Cycles, 18(1), 1-27.

Lehmann, S., Funck, D., Szabados, L., \& Rentsch, D. (2010). Proline metabolism and transport in plant development. Amino Acids, 39(4), 949-962.

Levitt, J. (1980). Responses of plants to environmental stresses. Volume I. Chilling, freezing and high temperature stresses. New York: Academic Press.

Li, X., Cai, J., Liu, F., Dai, T., Cao, W., \& Jiang, D. (2014). Spring freeze effect on wheat yield is modulated by winter temperature fluctuations: Evidence from meta-analysis and simulating experiment. Journal of Agronomy and Crop Science, 201(4), 288-300.

Li, X., Cai, J., Liu, F., Zhou, Q., Dai, T., Cao, W., et al. (2015). Wheat plants exposed to winter warming are more susceptible to low temperature stress in the spring. Plant Growth Regulation, 77(1), 11-19.

Liang, X., Zhang, L., Natarajan, S. K., \& Becker, D. F. (2013). Proline mechanisms of stress survival. Antioxidants \& Redox Signaling, 19(9), 998-1011.

Limin, A. E., \& Fowler, D. B. (2006). Low-temperature tolerance and genetic potential in wheat (Triticum aestivum $\mathrm{L}$ ): Response to photoperiod, vernalization, and plant development. Planta, 224(2), 360-366.

Lissarre, M., Ohta, M., Sato, A., \& Miura, K. (2010). Cold-responsive gene regulation during cold acclimation in plants. Plant Signaling \& Behavior, 5(8), 948-952.

Livingston, D. P. I., Tuong, T. D., Isleib, T. G., \& Murphy, J. P. (2016). Differences between wheat genotypes in damage from freezing temperatures during reproductive growth. European Journal of Agronomy, 74, 164-172.

Lobell, D. B., \& Gourdji, S. M. (2012). The influence of climate change on global crop productivity. Plant Physiology, 160, 1686-1697.

Loescher, W. H., Tyson, R. H., Everard, J. D., Redgwell, R. J., \& Bieleski, R. L. (1992). Mannitol synthesis in higher plants: Evidence for the role and characterization of a NADPH-dependent 
mannose 6-phosphate reductase. Plant Physiology, 98, $1396-1402$.

Machakova, I., Hanisova, A., \& Krekule, J. (1989). Levels of ethylene, ACC, MACC, ABA and proline as indicators of cold hardening and frost resistance in winter wheat. Physiologia Plantarum, 76, 603-607.

Marcellos, H., \& Single, W. V. (1975). Temperatures in wheat during radiation frost. Australian Journal of Experimental Agriculture, 15(77), 818-822.

March, T., Knights, S., Biddulph, B., Ogbonnaya, F., Maccallum, R., \& Belford, R. (2015). The GRDC National Frost Initiative, Vol 2015.

Mayer, R. R., Cherry, J. H., \& Rhodes, D. (1990). Effects of heat shock on amino acid metabolism of cowpea cells. Plant Physiology, 94, 796-810.

Mazzucotelli, E., Tartari, A., Cattivelli, L., \& Forlani, G. (2006). Metabolism of gamma-aminobutyric acid during cold acclimation and freezing and its relationship to frost tolerance in barley and wheat. Journal of Experimental Botany, 57(14), 3755-3766.

Meijer, H. J., \& Munnik, T. (2003). Phospholipid-based signaling in plants. Annual Review of Plant Biology, 54, 265-306.

Miura, K., \& Furumoto, T. (2013). Cold signaling and cold response in plants. International Journal of Molecular Sciences, 14(3), 5312-5337.

Miura, K., \& Tada, Y. (2014). Regulation of water, salinity, and cold stress responses by salicylic acid. Frontiers in Plant Science, 5, 4.

Monroy, A. F., Dryanova, A., Malette, B., Oren, D. H., Ridha Farajalla, M., Liu, W., et al. (2007). Regulatory gene candidates and gene expression analysis of cold acclimation in winter and spring wheat. Plant Molecular Biology, 64(4), 409-423.

Morgan, J. (1992). Osmotic components and properties associated with genotypic differences in osmoregulation in wheat. Functional Plant Biology, 19(1), 67-76.

Munnik, T., \& Vermeer, J. E. M. (2010). Osmotic stress-induced phosphoinositide and inositol phosphate signalling in plants. Plant, Cell and Environment, 33(4), 655-669.

Nahar, K., Hasanuzzaman, M., \& Fujita, M. (2016). Roles of osmolytes in plant adaptation to drought and salinity. In N. Iqbal, R. Nazar, \& N. A. Khan (Eds.), Osmolytes and plants acclimation to changing environment: Emerging omics technologies (pp. 37-68). India: Springer.

Narayanan, S., Prasad, P. V., \& Welti, R. (2016a). Wheat leaf lipids during heat stress: II. Lipids experiencing coordinated metabolism are detected by analysis of lipid co-occurrence. Plant, Cell and Environment, 39(3), 608-617.

Narayanan, S., Tamura, P. J., Roth, M. R., Prasad, P. V., \& Welti, R. (2016b). Wheat leaf lipids during heat stress: I. High day and night temperatures result in major lipid alterations. Plant, Cell and Environment, 39(4), 787-788.

Natera, S. H. A., Hill, C. B., Rupasinghe, T. W. T., \& Roesner, U. (2016). Salt-stress induced alterations in the root lipidome of two barley genotypes with contrasting responses to salinity. Functional Plant Biology, 43(2), 207-219.

Nishiyama, I. (1984). Climatic influence on pollen formation and fertilization. In S. Tsunoda \& N. Takahashi (Eds.), Biology of rice (pp. 153-171). Amsterdam: Elsevier.

Oliver, S. N., Van Dongen, J. T., Alfred, S. C., Mamun, E. A., Zhao, X., Saini, H. S., et al. (2005). Cold-induced repression of the rice anther-specific cell wall invertase gene OSINV4 is correlated with sucrose accumulation and pollen sterility. Plant, Cell and Environment, 28(12), 1534-1551.

Ouellet, F., \& Charron, J. B. (2013). Cold acclimation and freezing tolerance in plants. Encyclopedia of Life Sciences. https://doi. org/10.1002/9780470015902.a0020093.
Pagter, M., \& Arora, R. (2013). Winter survival and deacclimation of perennials under warming climate: Physiological perspectives. Physiologia Plantarum, 147(1), 75-87.

Palta, J. P., \& Weiss, L. S. (1993). Ice formation and freezing injury: An overview on the survival mechanisms and molecular aspects of injury and cold acclimation in herbaceous plants. In P. H. Li \& L. Christersson (Eds.), Advances in plant cold hardiness (pp. 143-176). Boca Raton (Florida): CRC Press.

Rapacz, M. (2002). Regulation of frost resistance during cold deacclimation and re-acclimation in oilseed rape. A possible role of PSII redox state. Physiologia Plantarum, 115(2), 236-243.

Rawlinson, C., Kamphuis, L. G., Gummer, J. P. A., Singh, K. P., \& Trengove, R. D. (2015). A rapid method for profiling of volatile and semi-volatile phytohormones using methyl chloroformate derivatisation and GC-MS. Metabolomics, 11(6), 1922-1933.

Ren, M., Phoon, C. K. L., \& Schlame, M. (2014). Metabolism and function of mitochondrial cardiolipin. Progress in Lipid Research, 55, 1-6.

Rerkasem, B. (1996). A general survey of the incidence of wheat sterility. In H. M. Rawson \& K. D. Subedi (Eds.), ACIAR Proceedings of the Sterility in wheat in subtropical Asia: extent, causes and solutions, Vol 72 (pp. 8-12). Pokhara (Nepal): LumJe Agricultural Research Centre.

Richards, R. A., Rebetzke, G. J., Watt, M., Condon, A. G., Spielmeyer, W., \& Dolferus, R. (2010). Breeding for improved water productivity in temperate cereals: Phenotyping, quantitative trait loci, markers and the selection environment. Functional Plant Biology, 37(2), 85-97.

Rimando, A. M., \& Perkins-Veazie, P. M. (2005). Determination of citrulline in watermelon rind. Journal of Chromatography A, 1078(1-2), 196-200.

Ruelland, E., Vaultier, M.-N., Zachowski, A., \& Hurry, V. (2009). Chapter 2: Cold signalling and cold acclimation in plants. In K. Jean-Claude \& D. Michel (Eds.), Advances in botanical research (Vol. 49, pp. 35-150). New York: Academic Press.

Sanchez, A., Shin, J., \& Davis, S. J. (2011). Abiotic stress and the plant circadian clock. Plant Signaling and Behaviour, 6(2), 223-231.

Satake, T., Nishiyama, I., Ito, N., \& Hayase, H. (1969). Male sterility caused by cooling treatment at the meiotic stage in rice plants. I. Methods of growing rice plants and inducing sterility in the phytotron. Japanese Journal of Crop Science, 38, 603-609.

Shavrukov, Y., Kurishbayev, A., Jatayev, S., Shvidchenko, V., Zotova, L., Koekemoer, F., et al. (2017). Early flowering as a drought escape mechanism in plants: How can it aid wheat production? Frontiers in Plant Science, 8, 950.

Shi, H., \& Chan, Z. (2014). Improvement of plant abiotic stress tolerance through modulation of the polyamine pathway. Journal of Integrative Plant Biology, 56(2), 114-121.

Slama, I., Abdelly, C., Bouchereau, A., Flowers, T., \& Savouré, A. (2015). Diversity, distribution and roles of osmoprotective compounds accumulated in halophytes under abiotic stress. Annals of Botany, 115(3), 433-447.

Smallwood, M., \& Bowles, D. J. (2002). Plants in a cold climate. Philosophical Transactions of the Royal Society B: Biological Sciences, 357(1423), 831-847.

Smith, A. R., \& Zhao, D. (2016). Sterility caused by floral organ degeneration and abiotic stresses in Arabidopsis and cereal grains. Frontiers in Plant Science, 7, 1503.

Steponkus, P. L., Uemura, M., \& Webb, M. S. (1993). A contrast of the cryostability of the plasma membrane of winter rye and spring oat-two species that widely differ in their freezing tolerance and plasma membrane lipid composition. In P. L. Steponkus (Ed.), Advances in low-temperature biology (Vol. 2, pp. 211-312). London: JAI Press.

Subedi, K. D., Floyd, C. N., \& Budhathoki, C. B. (1998a). Cool temperature-induced sterility in spring wheat (Triticum aestivum L.) 
at high altitudes in Nepal: Variation among cultivars in response to sowing date. Field Crops Research, 55(1-2), 141-151.

Subedi, K., Gregory, P., Summerfield, R., \& Gooding, M. (1998b). Cold temperatures and boron deficiency caused grain set failure in spring wheat (Triticum aestivum L.). Field crops research, $57(3), 277-288$.

Subedi, K. D., Gregory, P. J., Summerfield, R. J., \& Gooding, M. J. (2000). Pattern of grain set in boron-deficient and cold-stressed wheat (Triticum aestivum L). The Journal of Agricultural Science, 134(1), 25-31.

Sumner, L. W., Amberg, A., Barrett, D., Beale, M. H., Beger, R., \& Daykin, C. A. (2007). Proposed minimum reporting standards for chemical analysis Chemical Analysis Working Group (CAWG) Metabolomics Standards Initiative (MSI). Metabolomics, 3(3), 211-221.

Sumner, L. W., Lei, Z., Nikolau, B. J., \& Saito, K. (2015). Modern plant metabolomics: Advanced natural product gene discoveries, improved technologies, and future prospects. Natural Product Reports, 32, 212-229.

Tomashow, M. F. (1999). Plant cold acclimation: Freezing tolerance genes and regulatory mechanisms. Annual Review of Plant Physiology and Plant Molecular Biology, 50, 571-599.

Travert, S., Valerio, L., Fouraste, I., Boudet, A. M., \& Teulieres, C. (1997). Enrichment in specific soluble sugars of two Eucalyptus cell-suspension cultures by various treatments enhances their frost tolerance via a noncolligative mechanism. Plant Physiology, 114, 1433-1442.

Uemura, M., Joseph, R. A., \& Steponkus, P. L. (1995). Cold acclimation of Arabidopsis thaliana - effect on plasma membrane lipid composition and freeze-induced lesions. Plant Physiology, 109, $15-30$.

Uemura, M., \& Steponkus, P. L. (1994). A contrast of the plasma membrane lipid composition of oat and rye leaves in relation to freezing tolerance. Plant Physiology, 104, 479-496.

Uemura, M., Warren, G., \& Steponkus, P. L. (2003). Freezing sensitivity in the $s f r 4$ mutant of Arabidopsis is due to low sugar content and is manifested by loss of osmotic responsiveness. Plant Physiology, 131, 1800-1807.

Upchurch, R. G. (2008). Fatty acid unsaturation, mobilization, and regulation in the response of plants to stress. Biotechnology Letters, 30(6), 967-977.

Vágújfalvi, A., Kerepesi, I., Galiba, G., Tischner, T., \& Sutka, J. (1999). Frost hardiness depending on carbohydrate changes during cold acclimation in wheat. Plant Science, 144(2), 85-92.

Valluru, R., Lammens, W., Claupein, W., \& Van den Ende, W. (2008). Freezing tolerance by vesicle-mediated fructan transport. Trends in Plant Science, 13(8), 409-414.
Van Meer, G., Voelker, D. R., \& Feigenson, G. W. (2008). Membrane lipids: Where they are and how they behave. Nature Reviews Molecular Cell Biology, 9, 112-124.

Vanková, R., Kosová, K., Dobrev, P., Vítámvás, P., Trávníčková, A., Cvikrová, M., et al. (2014). Dynamics of cold acclimation and complex phytohormone responses in Triticum monococcum lines G3116 and DV92 differing in vernalization and frost tolerance level. Environmental and Experimental Botany, 101, 12-25.

Verslues, P. E., Agarwal, M., Katiyar-Agarwal, S., Zhu, J., \& Zhu, $\mathrm{J}-\mathrm{K}$. (2006). Methods and concepts in quantifying resistance to drought, salt and freezing, abiotic stresses that affect plant water status. Plant Journal, 45, 523-539.

Vigh, L., Horvath, L. I., Horvath, I., Dudits, D., \& Farkas, T. (1979). Protoplast plasmalemma fluidity of hardened wheats correlates with frost resistance. FEBS Letters, 107(2), 291-294.

Vitamvas, P., \& Prasil, I. T. (2008). WCS120 protein family and frost tolerance during cold acclimation, deacclimation and reacclimation of winter wheat. Plant Physiology and Biochemistry, 46(11), 970-976.

Wanner, L. A., \& Junttila, O. (1999). Cold-induced freezing tolerance in Arabidopsis. Plant Physiology, 120, 391-400.

Welti, R., Li, W., Li, M., Sang, Y., Biesiada, H., Zhou, H., et al. (2002). Profiling membrane lipids in plant stress responses. Journal of Biological Chemistry, 277(35), 31994-32002.

Welti, R., Shah, J., Li, W., Li, M., Chen, J., Burke, J. J., et al. (2007). Plant lipidomics: Discerning biological function by profiling plant complex lipids using mass spectrometry. Frontiers in Bioscience, 12, 2494-2506.

Zadoks, J. C., Chang, T. T., \& Konzak, C. F. (1974). A decimal code for the growth stages of cereals. Weed Research, 14, 415-421.

Zhang, W., Yu, L., Zhang, Y., \& Wang, X. (2005). Phospholipase D in the signaling networks of plant response to abscisic acid and reactive oxygen species. Biochimica et Biophysica Acta, 1736(1), $1-9$.

Zheng, B., Chapman, S. C., Christopher, J. T., Frederiks, T. M., \& Chenu, K. (2015). Frost trends and their estimated impact on yield in the Australian wheatbelt. Journal of Experimental Botany, 66(12), 3611-3623.

Zheng, G., Li, L., \& Li, W. (2016). Glycerolipidome responses to freezing- and chilling-induced injuries: Examples in Arabidopsis and rice. BMC Plant Biology, 16, 70.

Publisher's Note Springer Nature remains neutral with regard to jurisdictional claims in published maps and institutional affiliations. 